\title{
THE
}

$9-1-1971$

\section{^-Nucleon Charge-Symmetry-Breaking Interaction. I. Separable Potentials}

\author{
K. Hartt \\ University of Rhode Island, hartt@uri.edu \\ E. Sullivan
}

Follow this and additional works at: https://digitalcommons.uri.edu/phys_facpubs

Terms of Use

All rights reserved under copyright.

\section{Citation/Publisher Attribution}

Hartt, K., \& Sullivan, E. (1971). ^-Nucleon Charge-Symmetry-Breaking Interaction. I. Separable Potentials. Physical Review D, 4(5), 1353-1366. doi: 10.1103/PhysRevD.4.1353

Available at: http://dx.doi.org/10.1103/PhysRevD.4.1353

This Article is brought to you for free and open access by the Physics at DigitalCommons@URI. It has been accepted for inclusion in Physics Faculty Publications by an authorized administrator of DigitalCommons@URI. For more information, please contact digitalcommons-group@uri.edu. 


\title{
$\Lambda$-Nucleon Charge-Symmetry-Breaking Interaction. I. Separable Potentials*
}

\author{
K. Hartt and E. Sullivan $\dagger$ \\ Department of Physics, University of Rhode Island, Kingston, Rhode Island 02881
}

(Received 8 September 1969; revised manuscript received 17 May 1971)

\begin{abstract}
The existence of a charge-symmetry-breaking (CSB) term in the lambda-nucleon $(\Lambda-N)$ interaction is shown to produce an admixture of a $T=1$ state to the predominant isospin ; $\sin$ glet state in ${ }_{\Lambda} \mathrm{H}^{3}$. The present analysis shows that a CSB term constructed to fit light hypernuclear and $\Lambda-p$ scattering data is poorly determined $(\sim 4 \%-25 \%$ of the total $\Lambda-p$ singlet and $\sim 4 \%-15 \%$ of the total $\Lambda-p$ triplet interaction), but is strongly correlated with the $\Lambda$-separation energy, $B_{\Lambda}$, from ${ }_{\Lambda} \mathrm{H}^{3}$, and that isospin mixing in ${ }_{\Lambda} \mathrm{H}^{3}$ can lead to significant adjustments of the deduced $\Lambda-N$ interaction strengths. The mixed-symmetry $T=0$ state in ${ }_{\Lambda} \mathrm{H}^{3}$. is seen to have a small effect. Data employed include $\Lambda-p$ total cross sections, $\sigma(\Lambda p)$, for total c.m. energy $E_{\text {c.m. }}<18 \mathrm{MeV}$, published values of $B_{\Lambda}$, and a theoretical determination by Herndon and Tang of the relative magnitudes of the $\Lambda-N$ potentials responsible for the mean binding and the ground-state splitting of ${ }_{\Lambda} \mathrm{He}^{4}$ and ${ }_{\Lambda} \mathrm{H}^{4}$. The two hypotheses that the spin, $I$, of ${ }_{\Lambda} \mathrm{He}^{4}$ is 0 and 1 are studied. Nonlocal separable, spin-dependent central interactions of the Yamaguchi form are used, and all range parameters in the $\Lambda-N$ interaction are constrained to be equal. A search of fits to $\sigma(\Lambda p)$ is made with intrinsic ranges in the interval 1.5 $\mathrm{F}$ to $2.3 \mathrm{~F}$. Self-consistent CSB potentials are found for $I=0$ and $I=1$. The deduced singlet $\Lambda-p$ CSB interaction is attractive in all cases, in disagreement with predictions of the $\mathrm{SU}_{3}$ particle-mixing model of Downs.
\end{abstract}

\section{INTRODUCTION}

$\Lambda$ hyperons interact differently with neutrons than with protons. This charge-symmetry-breaking (CSB) effect is directly observed in the greater $\Lambda$ separation energy in ${ }_{\Lambda} \mathrm{He}^{4}$ than in ${ }_{\Lambda} \mathrm{H}^{4}$, the present experimental value for this difference being given $b^{1}$

$$
\begin{aligned}
\Delta \boldsymbol{B}_{\Lambda} & =B_{\Lambda}\left({ }_{\Lambda} \mathrm{He}^{4}\right)-B_{\Lambda}\left({ }_{\Lambda} \mathrm{H}^{4}\right) \\
& =0.28 \pm 0.07 \mathrm{MeV} .
\end{aligned}
$$

In 1964, Dalitz and von Hippel found that the superposition of different isospin states both in the physical $\Lambda$ 's and the physical pions, which they deduced from the Coleman-Glashow relation for electromagnetic mass splitting, could contribute substantially to CSB. ${ }^{2,3}$ With such isospin mixing, the isospin-forbidden exchange of a single pion between a $\Lambda$ and a nucleon could occur, and would contribute with the right order of magnitude and sign to $\Delta B_{\Lambda}$. The analysis by Downs, including particle mixing of vector mesons, pseudoscalar mesons, and baryons, then showed that vectormeson exchange also could contribute significantly to CSB, and that pion- $\eta$ mixing effects were reduced by cancellations. ${ }^{4}$ Downs constructed a oneboson-exchange potential model for his analysis, which employed the octet model of $\mathrm{SU}_{3}$ to provide relationships between many of the unknown couplings in his theory.

It is of interest to study and delimit the spin dependence and magnitude of the CSB lambda- nucleon $(\Lambda-N)$ potential as deduced from experiment. Although it is perhaps premature to expect a direct test thereby of Downs's model, a phenomenological analysis of light hypernuclei and lambdaproton $(\Lambda-p)$ scattering might provide some useful information. Consistency checks between the assumptions of the model and the values assumed for some of the experimentally uncertain mesonbaryon couplings ${ }^{5,6}$ can be of value. Downs performed one such check in showing that of the two possible values of the coupling constant $g_{\Lambda \Lambda \omega}, 6$ and -5 , the first was more clearly compatible with the size of CSB apparently needed for $\Delta B_{\Lambda} \cdot{ }^{4}$ In the phenomenological analysis which he made upon the four-body hypernuclei, the tensor part of the CSB potential, which has zero expectation value in relative $S$ states, was not considered. ${ }^{4}$

The central spin-isospin dependence of Downs's CSB interaction has the form $-\tau_{3}(N) \vec{\sigma}(N) \cdot \vec{\sigma}(\Lambda)$ for pseudoscalar-meson exchange, and $-\tau_{3}(N)$ $\times[A+B \vec{\sigma}(N) \cdot \vec{\sigma}(\Lambda)]$ for vector-meson exchange. The combination of all his central potential terms leads to a substantial spin-independent part. This is easily seen by computing the total volume integral of the spin-independent and spin-dependent contributions from his Eqs. (19) and (20). ${ }^{4}$ Since the vector mesons are heavier than the pseudoscalar mesons considered, a hard core in the CSB interaction, if present, would tend to reduce the spinindependent part in a different manner than the spin-dependent part. This is seen in Fig. 1, in which the total volume integral of the CSB $\Lambda-p$ 
potential is plotted as a function of a hard-core radius $R$. For a Yukawa potential, $e^{-\mu r} / \mu r$, the corresponding volume integral is just

$$
J=\frac{4 \pi}{\mu^{3}} e^{-\mu R}(1+\mu R) .
$$

The summed volume integrals for the complete central part of Downs's CSB $\Lambda-N$ interaction have the form $-\tau_{3}(N)[C+D \vec{\sigma}(\Lambda) \cdot \vec{\sigma}(N)]$, and $C$ and $D$ are plotted. A reduction of $C / D$ with increase in $R$ occurs at $R \geqslant 0.3 \mathrm{~F}$ and is clearly rather gradual. A feature particularly worth noting is that the singlet $\Lambda-p$ volume integral, $C-3 D$, is always negative. Since the tensor interaction vanishes in the singlet state, we conclude that the Downs model predicts a repulsive singlet $\Lambda-p$ interaction. This conclusion holds for extensive variations of the $\mathrm{SU}_{3}$ couplings we made about the experimental values quoted in Ref. 4 . The sign of $C-3 D$ becomes positive (attractive CSB singlet $\Lambda-p$ ) only when the isospin mixing in the physical lambda is more than 16 times smaller than the theoretical estimates made in Ref. 4.

The purpose of this paper is to use two-, three-, and four-baryon hypernuclear and scattering data in determining the parameters of a CSB singlet and triplet central $\Lambda-N$ interaction. We find a class of such potentials with systematic relationships between our CSB parameters and the range of experimental possibilities for low-energy $\Lambda-p$ scattering length as well as several quoted values

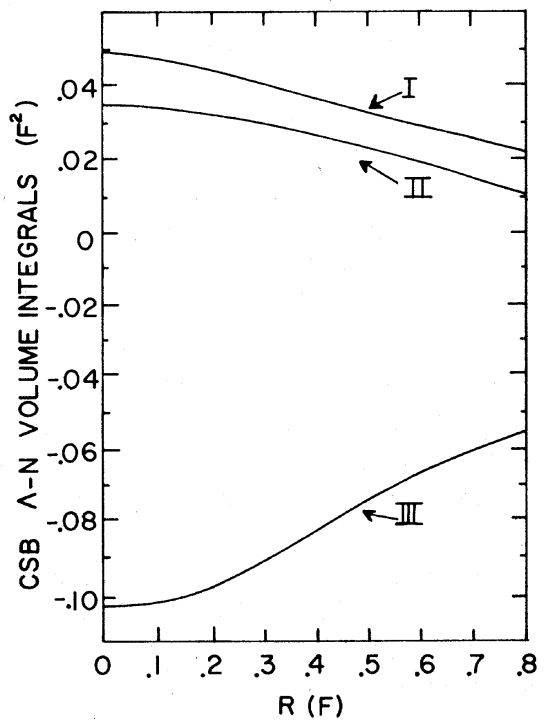

FIG. 1. Volume integrals of the central CSB interaction given in Ref. 4, as a function of hard-core radius, $R$. Curve $I$ is the spin-independent part; curve $I I$ is the factor of $\vec{\sigma}(\Lambda) \cdot \vec{\sigma}(N)$; curve III is the singlet volume integral. These are denoted in Sec. I, respectively, by $C$, $D$, and $C-3 D$. Assumed value of $g_{\Lambda \Lambda \omega}$ is 6 . for the $\Lambda$-separation energy from ${ }_{\Lambda} \mathrm{H}^{3}$, which we label as $B_{\Lambda}$. Because of the factor $\tau_{3}(N)$ in the $\Lambda-N$ interaction, isospin is not a good quantum number in hypernuclei. In particular, the CSB spin dependence produces an isospin mixing in ${ }_{\Lambda} \mathrm{H}^{3}$. An evaluation is made here of this effect upon $\Lambda-N$ potential parameters. The possibility that the spin of ${ }_{\Lambda} \mathrm{He}^{4}$ might be 1 also is considered.

The CSB potential produces a greater energy difference than the value for $\Delta B_{\Lambda}$ of Eq. (1), since the value of $\Delta B_{\Lambda}$ would be negative in the absence of CSB. This is because of the Coulomb energy associated with compression of the $\mathrm{He}^{3}$ core, and the differences in the form factors of the nuclear cores, $\mathrm{H}^{3}$ and $\mathrm{He}^{3} .^{2,4}$ Upon including these effects in their solutions for three- and four-body hypernuclei, Herndon and Tang have found a greater CSB contribution to the $\Lambda$-separation energy in ${ }_{\Lambda} \mathrm{He}^{4}$ than in ${ }_{\Lambda} \mathrm{H}^{4}$ by the amount $\Delta \boldsymbol{B}_{4}=0.54 \mathrm{MeV} .{ }^{7}$ They assumed a spin of 0 for ${ }_{\Lambda} \mathrm{He}^{4}$.

The spin dependence of the CSB interaction has been discussed by Downs and Phillips. ${ }^{8}$ In a phenomenological analysis which estimated $\Delta B_{4}$ at $0.75 \mathrm{MeV}$, they obtained a $\Lambda-N \mathrm{CSB}$ interaction which contributed significantly to the elastic $\Lambda-p$ scattering cross section, $\sigma(\Lambda p)$. They considered the more general spin dependence in the central CSB interaction, $A+B \vec{\sigma}(\Lambda) \cdot \vec{\sigma}(N)$, pointing out that only the linear combination $A+3 B$ is determined by $\Delta B_{4}$, but that the large values of $\sigma(\Lambda p)$ seemed consistent with the dominance of $A$. In deducing $\Lambda-N$ CSB and charge-symmetric (CS) hard-core potentials, HT assumed the case of $A=0$. Both DP and HT used $\sigma(\Lambda p)$ data as a final test of potentials constructed so as to be consistent with hyperfragment binding energies.

Dalitz recently suggested using a variety of $\Lambda-p$ effective-range parameters in theoretical investigations of hypernuclei, inasmuch as the data appear to support many sets of these parameters nearly equally well. ${ }^{9}$ parallel course, given adequate $\Lambda-p$ scattering data, is to determine the parameters in $\Lambda-p$ potentials for good $\chi^{2}$ fits, and then to specify the CSB interaction by requiring consistency with hyperfragment binding energies. This is feasible provided that the computer time spent determining binding energies given by the large variety of potentials tested is not prohibitive. Such a procedure is employed in this paper. It has the advantage of first meeting the most stringent requirement, fitting $\sigma(\Lambda p)$, thereby enabling a good over-all data fit. We employ nonlocal separable (NLS) potentials, well known for their amenability to efficient analysis of three-body systems. ${ }^{10}$ Our solution of the ${ }_{\Lambda} \mathrm{H}^{3}$ eigenvalue problem is discussed in Sec. II.

The data for $\Lambda-p$ scattering tend to support many 
different interpretations as to singlet and triplet scattering lengths and intrinsic ranges, ${ }^{11,12}$ requiring an extensive search of $\Lambda-p$ potentials. However, the potential parameters we find, although by no means unique, enable a number of conclusions to be drawn concerning the nature of CS and CSB potentials, within the limitations of the assumptions we make. Shown in Table $I$ is a combination of the recently published $\Lambda-p$ data of Alexander et al. ${ }^{11}$ and Secci-Zorn et al. ${ }^{12}$ at six points with total c.m. energy $E_{\text {c.m. }}<18 \mathrm{MeV}$. As discussed in Sec. III, the resulting data are smooth enough to allow fits by $S$-wave interactions at a confidence level of 0.61 .

For $B_{\Lambda}$ we use the published values of $0.05 \mathrm{MeV}^{13}$ and $0.25 \mathrm{MeV}^{14}$ and we also include results for the $0.17 \mathrm{MeV}$ value quoted by $\mathrm{HT}$. The final datum employed in this paper consists of a theoretical estimate by $\mathrm{HT}$ of the relative magnitudes of the effective CSB and CS interactions in the mirror hypernuclei ${ }_{\Lambda} \mathrm{He}^{4}$ and ${ }_{\Lambda} \mathrm{H}^{4}$, expressed in terms of a dimensionless parameter, $\Delta . \Delta$ is defined and evaluated later in this section.

Although it has been suggested that CSB effects cancel in ${ }_{\Lambda} \mathrm{H}^{3}, 2,8,15$ this is strictly true only when the possibility of isospin mixing is not considered. As shown in Sec. II, CSB produces an admixture of a $T=1$ state to the predominant isospin single ${ }^{+}$ state in ${ }_{\Lambda} \mathrm{H}^{3}$. In some cases, our CSB term is large enough to lead to significant adjustments of the $\Lambda-N$ interaction strengths upon inclusion of isospin mixing in the theoretical description of ${ }_{\Lambda} \mathrm{H}^{3}$.

Recent studies of the $T=0$ mixed-symmetry state $\left(S^{\prime}\right)$ in ${ }_{\Lambda} \mathrm{H}^{3}$ have shown that such a state leads to a reduction of the spin dependence of the CS interaction $^{16}$ and that the percentage $S^{\prime}$ state increases upon the introduction of short-range $\Lambda-N$ and $N-N$ repulsions. ${ }^{17}$ As seen in Sec. II, the $S^{\prime}$ state that we compute has a smaller effect upon $B_{\Lambda}$ than the dominant $T=1$ state.

The CSB interaction operator can be written as

$W_{\mathrm{CSB}}(\Lambda, N)=-\frac{\lambda_{B}}{2 \mu_{N} \Lambda} \tau_{3}(N)[\alpha+\vec{\sigma}(\Lambda) \cdot \vec{\sigma}(N)] W_{p}(\Lambda, N)$,

where $\lambda_{B}$ is the over-all interaction strength, $\mu_{N} \Lambda$ is the reduced mass of $N$ and $\Lambda, W_{p}$ is the spinand isospin-independent part of the interaction, and $\tau_{3}(N)$ is a Pauli operator in isospin space. Without making the restriction $\alpha=0$, we arrive at bounds of interaction strengths and intrinsic ranges of the CS and CSB interactions that are consistent with the following assumptions:

(a) The $\Lambda-N$ interaction is represented by twobody NLS spin-dependent central potentials of the Yamaguchi form. ${ }^{18}$ Then, for example, $W_{p}\left(\Lambda, N_{i}\right)$
TABLE I. Combined results of Alexander et al. and Secci-Zorn et al. Total c.m. energies are listed. They correspond to the average $\Lambda$ momentum within each momentum interval as measured by Secci-Zorn et al.

\begin{tabular}{cc}
\hline $\begin{array}{l}E_{\mathrm{c} . \mathrm{m} .} \\
(\mathrm{MeV})\end{array}$ & $\begin{array}{c}\sigma(\Lambda p) \\
(\mathrm{mb})\end{array}$ \\
\hline 3.7 & $211.0 \pm 31.0$ \\
5.6 & $154.3 \pm 22.0$ \\
7.7 & $146.0 \pm 15.0$ \\
10.5 & $101.0 \pm 10.7$ \\
13.0 & $83.0 \pm 7.3$ \\
17.6 & $52.4 \pm 7.7$ \\
\hline \hline
\end{tabular}

would be written in momentum space as

$$
\begin{aligned}
& W_{p}\left(\Lambda, N_{i}\right) \Psi\left(P_{\Lambda}, \ldots, P_{N_{i}}, \ldots\right) \\
& \quad=-g(P) \int d^{3} P^{\prime} d^{3} P_{c}^{\prime} g\left(P^{\prime}\right) \Psi\left(P_{\Lambda}^{\prime}, \ldots, P_{N_{i}}^{\prime}, \ldots\right) \delta\left(P_{c}^{\prime}-P_{c}\right),
\end{aligned}
$$

where $\boldsymbol{P}$ and $P_{c}$ are the relative and c.m. momenta of $\Lambda$ and the $i$ th nucleon. The Yamaguchi kernel, $g(P)=\left(P^{2}+\beta^{2}\right)^{-1}$, has the effect of projecting onto the $S$ state of the relative motion of $\Lambda$ and $N_{i}$. CS potentials of this form have been employed by Hetherington and Schick ${ }^{10}$ in their study of $\Lambda-d$ scattering and ${ }_{\Lambda} \mathrm{H}^{3}$. Although NLS interactions in states of higher angular momentum can be constructed in a similar fashion, we make the assumption here that their contribution in explaining the data we use is small enough to be neglected.

(b) The $n-p$ interaction is approximated by singlet-even and triplet-even Yamaguchi kernels, whose range parameters and interaction strengths are determined by effective-range theory. These parameters are listed in Table II. The relatively weaker odd-state $n-p$ interactions are assumed to have negligible effect in these calculations.

(c) The intrinsic range, $b=3 / \beta$, is taken to be the same for all CSB and CS $\Lambda-N$ interaction terms. The singlet and triplet $\Lambda-p$ interaction strengths and $b$ are assigned a range of values obtained by fitting $\sigma(\Lambda p)$ for c.m. energies under $18 \mathrm{MeV}$.

(d) The tensor $\Lambda-N$ and $N-N$ interactions are represented by effective central triplet terms.

(e) A completely self-consistent treatment of two-, three-, and four-baryon data would require solving the four-body problems for ${ }_{\Lambda} \mathrm{He}^{4}$ and ${ }_{\Lambda} \mathrm{H}^{4}$. Although this is not done here, we assume that information about the four-body systems still is utilized in the form of a dimensionless parameter, $\Delta$, the value of which is inferred from the calculations of HT. $\Delta$ is chosen to represent the relative strength of the perturbation in the $\Lambda-N$ potential causing the splitting of four-baryon binding energies. Let $\lambda_{n p p}=$ spin-averaged $\Lambda-N$ interaction 
strength in ${ }_{\Lambda} \mathrm{He}^{4}$, and let $\lambda_{n n p}=$ spin-averaged $\Lambda-N$ interaction strength in ${ }_{\Lambda} \mathrm{H}^{4}$. Then we define

$$
\Delta=\frac{\lambda_{n p p}-\lambda_{n n p}}{\lambda_{n p p}+\lambda_{n n p}} .
$$

The ratio of the associated energies, $\frac{1}{2}\left(\Delta B_{4}\right) /($ average four-baryon $\Lambda$-separation energy), is 0.13 . $\Delta$ is necessarily appreciably smaller than 0.13 because the average $\Lambda-N$ potential must overcome kinetic energies as well as provide binding. The spin-averaged interaction strengths for the $\Lambda$-particle interacting with the nuclear cores are easily seen to be

$$
\begin{array}{ll}
\text { Spin 0: } & \lambda_{n p p}=\frac{1}{2}\left(\lambda_{t}+\lambda_{s}\right)+\frac{1}{3}(3+\alpha) \lambda_{B}, \\
\text { Spin 1: } & \lambda_{n p p}=\frac{1}{6}\left(5 \lambda_{t}+\lambda_{s}\right)+\frac{1}{3}(\alpha-1) \lambda_{B}, \\
\text { Spin 0: } & \lambda_{n n p}=\frac{1}{2}\left(\lambda_{t}+\lambda_{s}\right)-\frac{1}{3}(3+\alpha) \lambda_{B},
\end{array}
$$

where the CS triplet and singlet $\Lambda-N$ interaction strengths are denoted by $\lambda_{t}$ and $\lambda_{s}$.

Following HT, we adopt the experimental value of 0 for the spin of ${ }_{\Lambda} \mathrm{H}^{4}$. However, as the spin, $I$, of ${ }_{\Lambda} \mathrm{He}^{4}$ appears to be unknown, we consider both the possibilities $I=0$ and $I=1$, given in (6a) and

(6b). The first case $(I=0)$ leads to

$$
\Delta=\frac{\frac{2}{3}(3+\alpha) \lambda_{B}}{\lambda_{t}+\lambda_{s}} .
$$

The second case $(I=1)$ yields

$$
\Delta=\frac{\frac{2}{3}(\alpha+1) \lambda_{B}+\frac{1}{3}\left(\lambda_{t}-\lambda_{s}\right)}{\lambda_{t}+\lambda_{s}},
$$

where the denominator in Eq. (7b) is taken to be the same as in Eq. (7a) with negligible error. Using potential $H$ of HT (they set $\alpha=0$ ) produces the value $\Delta=0.0099 \pm 0.0012$, which is taken as a final piece of data to be fitted by our phenomenological $\Lambda-N$ interaction. $^{19}$ The quoted error in $\Delta$

TABLE II. Yamaguchi $n-p$ potential parameters.$^{a}$

\begin{tabular}{ccccc}
\hline \hline & $\beta^{\mathrm{b}}$ & $\lambda$ & $\begin{array}{c}\text { Scattering } \\
\text { length } \\
\text { State- }\end{array}$ & $\begin{array}{c}\text { Effective } \\
\text { range } \\
(\mathrm{F})\end{array}$ \\
\hline $\begin{array}{c}\text { Singlet } \\
\text { even }\end{array}$ & 1.163 & 0.1486 & $-23.714 \pm 0.013$ & $2.704 \pm 0.095$ \\
$\begin{array}{c}\text { Triplet } \\
\text { even }\end{array}$ & 1.389 & 0.3696 & $5.425 \pm 0.005$ & $1.763 \pm 0.005^{\mathrm{c}}$ \\
\hline \hline
\end{tabular}

${ }^{a}$ For notation see Ref. 18. Effective-range parameters are taken from T. Houk and R. Wilson, Rev. Mod. Phys. 39, 546 (1967); ibid. 40, 672(E) (1968).

${ }^{b}$ Units are chosen such that $\hbar=c=1$.

${ }^{c}$ This is the mixed effective range, $\rho(0,-\epsilon)$. The value of $\rho(0,0)=r_{0 t}$ which is consistent with this triplet potential is given by $r_{0 t}=1.788 \mathrm{~F}$. The deuteron binding energy computed with these parameters is $2.2247 \mathrm{MeV}$. is associated with the experimental errors of the four-baryon energies. ${ }^{1}$

Although $\Delta$ is not an experimental number, there is a fair degree of consistency in its value obtained from the potentials of HT. The potentials introduced in Ref. 7 yield values of $\Delta$ in the range 0.0091 to 0.014 . The value 0.0099 should probably be considered a lower bound of the $\Delta$ expected with our potentials. The $0.6-\mathrm{F}$ hard core employed by HT creates larger kinetic energies with correspondingly sizeable cancellations than would be expected in the model containing no short-range repulsions that we use. In order to observe the effects of a plausible increase of $\Delta$, we have chosen the cases $\Delta=10^{-2}$ and $\Delta=1.5 \times 10^{-2}$. Strictly speaking, Coulomb effects in the structures of the nuclear cores $\mathrm{H}^{3}$ and $\mathrm{He}^{3}$ should be assessed in relation to our model. However, our analysis does not depend so sensitively upon $\Delta$ as to demand more precise estimates of these effects here. While we assume the same numerical value of $\Delta$ for the case $I=1$, the mechanism leading to a positive $\Delta$ as given in Eq. (7b) is clearly different. There are in addition possible isospin-mixing effects in the four-baryon systems, which are not treated by HT. Although we expect these to make a comparatively smaller contribution to $\boldsymbol{\Delta} \boldsymbol{B}_{4}$, they should be included in a more complete calculation.

Our potentials contain five independent parameters, an intrinsic range $b$, and singlet and triplet CS and CSB strengths. Fitting the $\Lambda-p$ interaction to $\sigma(\Lambda p)$ leaves only two independent parameters, determined uniquely by $B_{\Lambda}$ and $\Delta$ as discussed in Sec. IV.

The assumptions (a) $-(e)$ which we make in this initial analysis certainly do not have an unqualified validity. The large intrinsic ranges $(b \approx 1.8-2.1 \mathrm{~F})$ required for best fits of $\sigma(\Lambda p)$ contrast with the shorter intrinsic ranges $(b \leqslant 1.5 \mathrm{~F})$ associated with known meson-exchange processes. This has frequently been taken as evidence for a $\Lambda-N$ repulsive core. ${ }^{11,20}$ Such repulsions, requiring at least a rank-2 nonlocal interaction, would be an important refinement of assumption (a), and will be discussed in the following paper. The $\Lambda-N$ tensor interaction ${ }^{21}$ acts more weakly in ${ }_{\Lambda} \mathrm{H}^{3}$ than in $\Lambda-p$ scattering, ${ }^{22}$ and hence cannot be represented by the same effective central triplet interaction in both these systems without introducing some error. Tensor interactions and short-range repulsions known to be in $N-N$ interaction ${ }^{23}$ should also be included. Three-body forces are predicted as a consequence of two-pion-exchange processes, but can be expected to be less effective in ${ }_{\Lambda} \mathrm{H}^{3}$ than in heavier hypernuclei. ${ }^{24}$

Two-pion exchange is accomplished by the isospin-conserving virtual process $\Lambda-\Sigma+\pi$, result- 
ing in an intermediate state wherein the $\Lambda$ hyperon has been converted into a $\Sigma$ hyperon. Potential models which take $\Lambda-\Sigma$ conversion into account through the inclusion of an explicit $\Sigma$ component in the wave function have been derived mesontheoretically ${ }^{25}$ and have also been discussed in phenomenological three-body analyses. ${ }^{15,26}$ The resulting two-channel formalism (TCF) permits the distinction to be made between component potentials causing $\Lambda-\Sigma$ conversion and those which do not. Reference to the $\Sigma$ component can be eliminated, resulting in the one-channel formalism (OCF), but an artifact which then appears is manybody forces. Even at low energies the resulting three-body $\Lambda N N$ interaction can be large. ${ }^{27}$ Mainly because of the Pauli exclusion principle, which restricts the available intermediate nucleon states, $\Lambda-\Sigma$ conversion is considerably suppressed in nuclear matter. ${ }^{27,28}$ In addition, the isospin-conserving $\Lambda-\Sigma$ conversion is significantly suppressed in ${ }_{\Lambda} \mathrm{He}^{5}$ and can therefore help account for the observed anomalously low $\Lambda$-separation energy in ${ }_{\Lambda} \mathrm{He}^{5} .^{16}$

A final qualification of our assumptions, then, is that the OCF used in this paper, with assumed two-body interaction, might lead to a deduced CSB interaction with different properties from those obtained in a TCF calculation.

\section{ISOSPIN MIXING IN ${ }_{\Lambda} \mathrm{H}^{3}$}

The total potential-energy operator is $V_{\text {tot }}$ $=V_{N N}(2,3)+V_{\Lambda N}(1,2)+V_{\Lambda N}(1,3)$, where $(1,2,3)$ denote $(\Lambda, N, N)$ coordinates, and $V_{\Lambda N}(i, j)$ is given by $V_{\Lambda N}(i, j)=U_{C S}(i, j)+W_{C S B}(i, j) . W_{C S B}$ is defined in Eq. (3), and the symbol $U$ is reserved in what follows for the CS $\Lambda-N$ potential.

The two linearly independent spin eigenvectors of $\sigma_{z}$ and $\sigma^{2}$ for the $\left(S, S_{z}\right)=\left(\frac{1}{2}, \frac{1}{2}\right)$ state are $\mathrm{e}^{29}$

$$
\begin{aligned}
&\left|\chi_{+}\right\rangle=\left(\frac{1}{6}\right)^{1 / 2}[\alpha(1) \alpha(2) \beta(3) \\
&+\alpha(1) \beta(2) \alpha(3)-2 \beta(1) \alpha(2) \alpha(3)],
\end{aligned}
$$

$$
\left|\chi_{-}\right\rangle=\left(\frac{1}{2}\right)^{1 / 2}[\alpha(1) \alpha(2) \beta(3)-\alpha(1) \beta(2) \alpha(3)],
$$

where $\alpha(i)$ and $\beta(i)$ are "spin up" and "spin down" states.

The effect of the $\tau_{3}(N)$ factor in $W_{\mathrm{CSB}}(\Lambda, N)$ is to connect the $n p$ isosinglet to the $n p$ isotriplet substates of the $\Lambda n p$ system. The meson-theoretic CSB potential and also our $W_{\mathrm{CSB}}(\Lambda, N)$ make no reference to the isospin quantum numbers of the physical $\Lambda$ particle. Hence the isospin mixing in the OCF ${ }_{\Lambda} \mathrm{H}^{3}$ wave function depends only in an indirect fashion upon the $\Lambda-\Sigma^{0}$ mixing, through the potential $W_{\text {CSB }}$, and is obtained formally by setting to zero the isospin of the $\Lambda$ particle and solving the Schrödinger equation. This is strictly a formal device and is not in contradiction with the $\Lambda-\Sigma^{0}$ mixing responsible in large part for $W_{\mathrm{CSB}}$. The present calculation, which does not include two explicit channels for $\Lambda$ and $\Sigma$ states, cannot distinguish between the CSB contribution from $\Lambda-\Sigma^{0}$ mixing and isospin mixing of exchanged mesons.

In our model, the total isospin of the ${ }_{\Lambda} \mathrm{H}^{3}$ wave function is determined by $n$ and $p$ and is expressed in terms of the symmetric triplet state $\left|\xi_{+}\right\rangle$corresponding to $\left(T, T_{3}\right)=(1,0)$ and the antisymmetric singlet state $\left|\xi_{-}\right\rangle$corresponding to $\left(T, T_{3}\right)=(0,0)$ :

$$
\begin{aligned}
& \left|\xi_{+}\right\rangle=\left(\frac{1}{2}\right)^{1 / 2}[\pi(2) \nu(3)+\nu(2) \pi(3)], \\
& \left|\xi_{-}\right\rangle=\left(\frac{1}{2}\right)^{1 / 2}[\pi(2) \nu(3)-\nu(2) \pi(3)],
\end{aligned}
$$

where $\pi(i)$ and $\nu(i)$ are proton and neutron states.

The most general wave function obeying the generalized Pauli exclusion principle is a linear combination of products of the states in Eqs. (6) and (7), which we denote by $|S, T\rangle$, where $S=+$ for $\left|\chi_{+}\right\rangle$, the spin state which is symmetric in $n$ and $p$, and $S=-$ for $\left|\chi_{-}\right\rangle$, which is antisymmetric. $T$ is the total isospin. These four states are coupled by $V_{\text {tot }}$ as follows:

$$
\begin{aligned}
V_{\text {tot }}|+, 0\rangle= & {\left[V_{t e}(2,3)+\frac{1}{2} U_{2}(1,3)+\frac{1}{2} U_{2}(1,2)\right]|+, 0\rangle-\frac{1}{4} \sqrt{3}\left[U_{\sigma}(1,3)-U_{\sigma}(1,2)\right]|-, 0\rangle } \\
& -\frac{1}{2}\left[W_{2}(1,3)-W_{2}(1,2)\right]|+, 1\rangle+\frac{1}{4} \sqrt{3}\left[W_{\sigma}(1,3)+W_{\sigma}(1,2)\right]|-, 1\rangle, \\
V_{\text {tot }}|-, 0\rangle= & {\left[V_{s o}(2,3)+\bar{U}(1,3)+\bar{U}(1,2)\right]|-, 0\rangle-\frac{1}{4} \sqrt{3}\left[U_{\sigma}(1,3)-U_{\sigma}(1,2)\right]|+, 0\rangle } \\
& +[\bar{W}(1,2)-\bar{W}(1,3)]|-, 1\rangle+\frac{1}{4} \sqrt{3}\left[W_{\sigma}(1,2)+W_{\sigma}(1,3)\right]|+, 1\rangle, \\
V_{\text {tot }}|-, 1\rangle= & {\left[V_{s e}(2,3)+\bar{U}(1,3)+\bar{U}(1,2)\right]|-, 1\rangle-\frac{1}{4} \sqrt{3}\left[U_{\sigma}(1,3)-U_{\sigma}(1,2)\right]|+, 1\rangle } \\
& +[\bar{W}(1,2)-\bar{W}(1,3)]|-, 0\rangle+\frac{1}{4} \sqrt{3}\left[W_{\sigma}(1,2)+W_{\sigma}(1,3)\right]|+, 0\rangle, \\
V_{\text {tot }}|+, 1\rangle= & {\left[V_{t o}(2,3)+\frac{1}{2} U_{2}(1,3)+\frac{1}{2} U_{2}(1,2)\right]|+, 1\rangle-\frac{1}{4} \sqrt{3}\left[U_{\sigma}(1,3)-U_{\sigma}(1,2)\right]|-, 1\rangle } \\
& -\frac{1}{2}\left[W_{2}(1,3)-W_{2}(1,2)\right]|+, 0\rangle+\frac{1}{4} \sqrt{3}\left[W_{\sigma}(1,3)+W_{\sigma}(1,2)\right]|-, 0\rangle,
\end{aligned}
$$


where subscripted $\backslash V^{\prime}$ 's denote $N$ - $N$ interactions, $t, s, e, o$ stand for triplet, singlet, even, odd, and $U_{2}=\frac{3}{2} U_{s}+\frac{1}{2} U_{t}, \bar{U}=\frac{3}{4} U_{t}+\frac{1}{4} U_{s}, U_{\sigma}=U_{s}-U_{t}$ (similarly for $W$ ). Equations (10), derived in a straightforward manner using spin- and isospinprojection operators, are a generalization of the case of symmetry mixing considered earlier by Bodmer. ${ }^{16}$ However, upon dropping the states $|-, 1\rangle$ and $|+, 1\rangle$, a discrepancy remains with Eqs.

(15) of Ref. 16. As pointed out by one of us earlier, ${ }^{30}$ this discrepancy could result in an overestimation of the effect of $|-, 0\rangle$ (associated with Bodmer's $S^{\prime}$ state) upon the interaction strength $\lambda=\frac{3}{4} \lambda_{s}+\frac{1}{4} \lambda_{t}$ for fixed $\Lambda$-separation energy, $B_{\Lambda}$. Here, $\lambda_{s}$ and $\lambda_{t}$ are interaction strengths associated with $U_{s}$ and $U_{t}$.

With use of Eqs. (10) and the assumed interactions, the Schrödinger equation for ${ }_{\Lambda} \mathrm{H}^{3}$ reduces to coupled one-dimensional integral equations in a fashion that is by now well known. ${ }^{10}$ These equations, in which $B_{\Lambda}$ is known and $\lambda^{-1}$ is the eigenvalue, are presented in the Appendix.

Two special cases are considered in this paper. The first is the coupling of the dominant $|+, 0\rangle$ state to $|-, 0\rangle$. This enables a direct comparison with the results of Ref. 16. As the effect of $|-, 0\rangle$ upon $\lambda$ is shown to be small, we neglect this state in studying the second special case, namely, the coupling of $|+, 0\rangle$ to the $T=1$ state $|-, 1\rangle$. As can be seen in Eqs. (10), such a coupling can be enhanced by a strong spin dependence of $W_{\mathrm{CSB}}$ and the strongly attractive $n-p$ singlet-even potential. Mainly because the triplet-odd $n-p$ interaction is not strongly attractive (and in fact is set equal to 0 in our model), the $|+, 1\rangle$ state would be expected to exert a smaller influence upon $\lambda$.

The importance of the $S^{\prime}$ state in ${ }_{\Lambda} \mathrm{H}^{3}$ depends upon whether its presence significantly affects the $\Lambda-N$ interaction parameters required to fit the experimental binding energy. As shown in Ref. 16, the parameter directly affected is the spin dependence of $U_{C S}$. This is proportional to the interaction strength, $\lambda_{\sigma}$, defined by

$$
\lambda_{\sigma}=\lambda_{s}-\lambda_{t} \text {. }
$$

It is more convenient to work with the dimensionless parameter

$$
S=\frac{1}{4} \sqrt{3} \lambda_{\sigma} / \lambda .
$$

Figure 2(a) shows the variation of $\lambda$ with $S$, with $B_{\Lambda}$ kept fixed. This is obtained by repeatedly solving the ${ }_{\Lambda} \mathrm{H}^{3}$ equations with different values of $S$. This plot has been put in dimensionless form by defining $G(S)=1-\lambda(S) / \lambda(0)$. Increasing $B_{\Lambda}$ from $0.05 \mathrm{MeV}$ to $0.3 \mathrm{MeV}$ increases $G(S)$ by a maximum of $2.5 \%$ for $S \leqslant 0.3$. Therefore, only the case $B_{\Lambda}=0.17 \mathrm{MeV}$ is shown. The dependence of $G(S)$ upon the $\Lambda-N$ intrinsic range is indicated by considering $b=1.5 \mathrm{~F}$, which corresponds to $2 \pi$ exchange, and the three other intrinsic ranges, $1.8 \mathrm{~F}$, 2.1 F, 2.3 F. For comparison, the result of Ref. 16 for $b=1.5 . \mathrm{F}$ is expressed in our dimensionless form and shown as the dashed curve in Fig. 2(a). The present estimate of the effect of the $S^{\prime}$ state upon the $\Lambda-N$ interaction is appreciably smaller than that predicted in Ref. 16, and for the large majority of spin dependences of our potentials, can be neglected.

By contrast, isospin mixing in ${ }_{\Lambda} \mathrm{H}^{3}$ shows a stronger effect than the $S^{\prime}$ state in changing the value of the spin-averaged CS interaction strength, $\lambda$, that is required to produce a given $B_{\Lambda}$. As seen in Eqs. (10), the relevant interaction parameter in the isospin coupling is the CSB spin-difference strength, $\epsilon_{\sigma}$, defined by

$$
\epsilon_{\sigma}=\epsilon_{s}-\epsilon_{t}
$$

where $\epsilon_{s}$ and $\epsilon_{t}$ are the singlet and triplet CSB
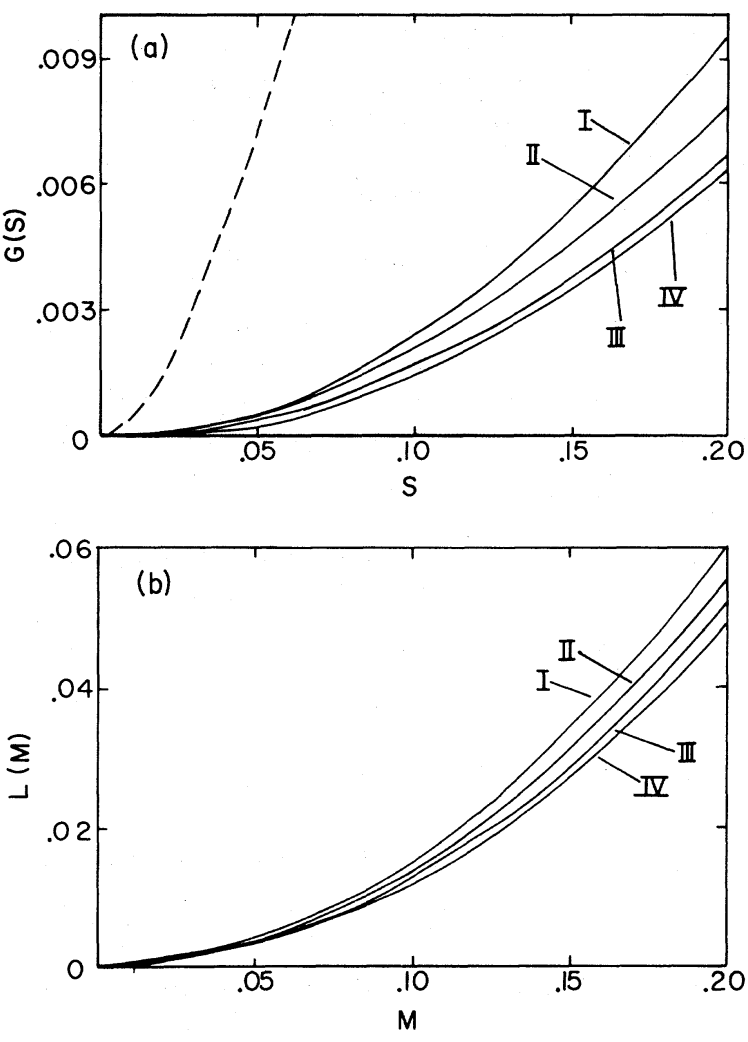

FIG. 2. (a) $G(S)$ curves representing effect of mixedsymmetry $S$ state in ${ }_{\Lambda} \mathrm{H}^{3}$, for $B_{\Lambda}=0.17 \mathrm{MeV}$ and the intrinsic ranges $b=1.5 \mathrm{~F}$ (I), $b=1.8 \mathrm{~F}$ (II), $b=2.1 \mathrm{~F}$ (III), and $b=2.3 \mathrm{~F}$ (IV). The dashed curve is for $b=1.5 \mathrm{~F}$ and is deduced from Ref. 16. (b) $L(M)$ curves representing effect of $T=1$ state in ${ }_{\Lambda} \mathrm{H}^{3}$, for $B_{\Lambda}=0.17 \mathrm{MeV}$ and the intrinsic ranges $b=1.5 \mathrm{~F}$ (I), $1.8 \mathrm{~F}$ (II), $2.1 \mathrm{~F}$ (III), and $2.3 \mathrm{~F}$ (IV). 
$\Lambda-N$ strengths. Again, it is more convenient to express results in dimensionless form. We define

$$
M=\frac{1}{4} \sqrt{3} \epsilon_{\sigma} / \lambda .
$$

In Fig. 2(b) we plot the dimensionless quantity $L(M)=1-\lambda(M) / \lambda(0)$. Unlike $G(S), L(M)$ is quite strongly dependent upon $B_{\Lambda}$. Keeping $B_{\Lambda}$ fixed, the ${ }_{\Lambda} \mathrm{H}^{3}$ Schrödinger equation is repeatedly solved for different assumed values of $M$. We show results for the same intrinsic ranges and $B_{\Lambda}$ as in Fig. 2(a).

Summarizing, upon inspection of Figs. 2(a) and 2(b), $\lambda$ is seen to depend more sensitively upon isospin mixing than upon symmetry $\left(S^{\prime}\right)$ mixing, when comparison is made at equal values of the dimensionless coupling parameters, $S$ and $M$.

\section{III. $\Lambda-p$ POTENTIALS}

The total $\Lambda-p$ elastic cross section for a c.m. momentum $k$ is given by

$$
\sigma(\Lambda p)=\frac{3 \pi}{k^{2}\left(1+\cot ^{2} \delta_{0 t}\right)}+\frac{\pi}{k^{2}\left(1+\cot ^{2} \delta_{0 s}\right)} .
$$

If we denote the $\Lambda-p$ singlet and triplet interaction strengths by $\lambda_{s x}$ and $\lambda_{t x}$, then a given $\Lambda-p$ potential is characterized by $\lambda_{s x}, \lambda_{t x}$ and intrinsic range, $b$. In a search of these parameters, the known analytic solutions for the singlet and triplet $S$-wave phase shifts $\delta_{0 s}$ and $\delta_{0 t}$, from a Yamaguchi potential ${ }^{18}$ are evaluated at the six energies of Table I. The $\chi^{2}$ function then is evaluated, $\chi^{2}$ being given by

$$
\chi^{2}=\sum_{i=1}^{6}\left(\frac{\sigma_{\exp }(i)-\sigma_{\mathrm{th}}(i)}{\Delta \sigma(i)}\right)^{2} .
$$

$\sigma_{\text {exp }}(i), \sigma_{\text {th }}(i)$, and $\Delta \sigma(i)$ are the measured and computed cross section and standard error at the $i$ th energy. The errors listed in Table I are reduced from those given in Refs. 11 and 12 as a consequence of the improved statistics obtained in combining the two sets of data. It should be noted that the data in Refs. 11 and 12 might not be entirely compatible, as these two groups use different methods of data analysis. However, in preliminary calculations, both sets of data appeared to produce similar results.

In our scans of fits to $\sigma(\Lambda p)$, we use the triplet and singlet scattering lengths, $a_{s}$ and $a_{t}$, as independent parameters, ${ }^{31}$ and the intrinsic range. Using a mesh of $0.1 \mathrm{~F}$, we search over the values $0 \leqslant-a_{s} \leqslant 7 \mathrm{~F}, 0 \leqslant-a_{t} \leqslant 3 \mathrm{~F}$ at the intrinsic ranges $1.3 \leqslant b \leqslant 2.5 \mathrm{~F}$. We denote by $\chi_{\text {min }}^{2}$ the minimum value of $\chi^{2}$ for the entire family of parameters scanned at a given intrinsic range. As seen in Fig. 3, although $\chi^{2}{ }_{\text {min }}$ reaches a least value at $b=2.1 \mathrm{~F}$, a valley of nearly equally good fits ex-

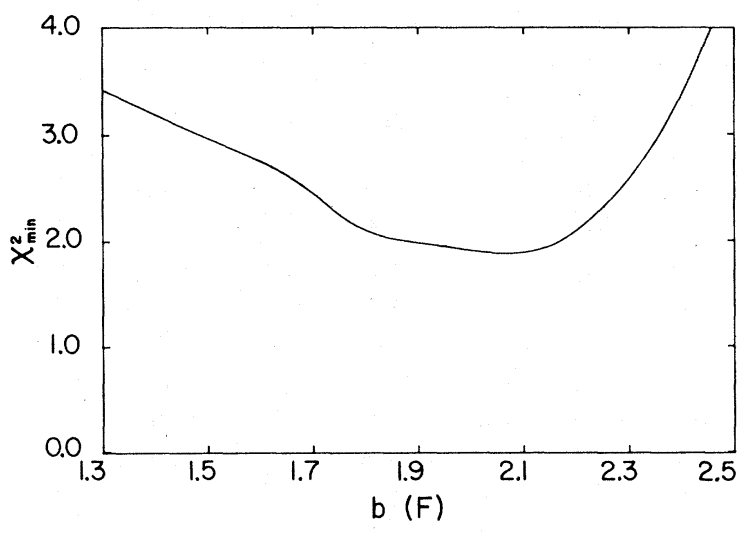

FIG. 3. Minimum value of $\chi^{2}$ as a function of intrinsic range, $b$. This curve represents complete scans of fits to $\sigma(\Lambda p)$ at intervals of $0.1 \mathrm{~F}$.

tends to $1.8 \mathrm{~F}$. In this paper, we give special attention to the intrinsic ranges $b=1.8$ and $2.1 \mathrm{~F}$ as representing best fits in addition to $b=2.3 \mathrm{~F}$ and $1.5 \mathrm{~F}$ just on the edges of the $\chi^{2}$ valley.

Contours for $\chi^{2}=2.8$ are shown in Fig. 4. Their interiors correspond to potential parameters for which $\chi^{2}-1.8 \leqslant 1$, where $\chi^{2}{ }_{\min }=1.8$ at $b=2.1 \mathrm{~F}$. In our search for solutions to the three- and fourbody data, we restrict ourselves to within the contours shown. For $b=1.5 \mathrm{~F}$ we find $\chi^{2}{ }_{\text {min }}=3.0$, but because of the special interest in $b=1.5 \mathrm{~F}$ as a probable upper limit associated with mechanisms for attractive $\Lambda-N$ potentials ${ }^{\mathbf{1 6}}$ we also include data fits at $b=1.5 \mathrm{~F}$ with $3.0 \leqslant \chi^{2} \leqslant 3.2$. These lie within the contour $\chi^{2}=3.3$ shown in Fig. 4. Clearly, the correlations between $a_{s}$ and $a_{t}$ observed by Alexander et al. persist in the present analysis. We obtain $124 \Lambda-p$ potentials which meet our $\chi^{2}$ criterion.

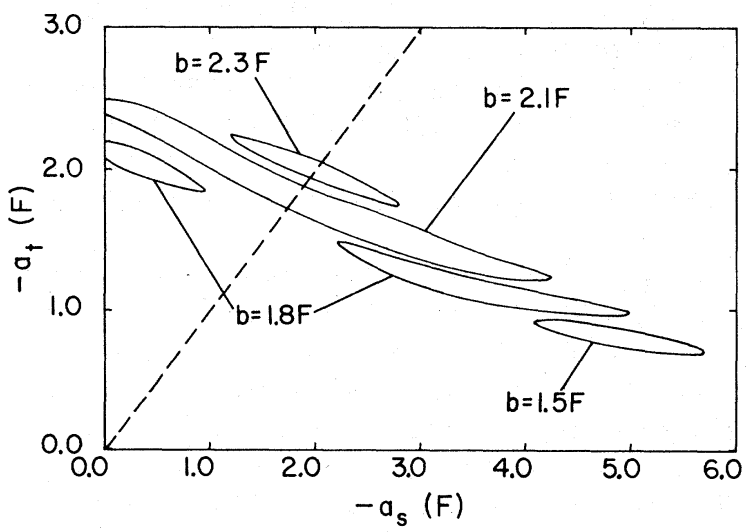

FIG. 4. Contours of constant $\chi^{2}$ for selected values of the intrinsic range, $b$, on the scattering-length plane. For $b=1.8,2.1$, and $2.3 \mathrm{~F}$ they enclose regions where $\chi^{2} \leq 2.8$. The contour marked $b=1.5 \mathrm{~F}$.encloses the region $\chi^{2} \leq 3.3$. 
TABLE III. Selected $\Lambda-p$ potentials.

\begin{tabular}{ccccccc}
\hline \hline & $b$ & & $-a_{s}$ & $-a_{t}$ & $r_{0 s}$ & $r_{0 t}$ \\
$(\mathrm{~F})$ & $(\mathrm{F})$ & $(\mathrm{F})$ \\
\hline 01 & 1.5 & 3.01 & 4.9 & 0.8 & 1.70 & 2.75 \\
02 & 1.5 & 3.20 & 4.2 & 0.9 & 1.74 & 2.61 \\
& & & & & & \\
03 & 1.8 & 2.35 & 3.5 & 1.2 & 2.21 & 3.00 \\
04 & 1.8 & 2.37 & 4.1 & 1.1 & 2.15 & 3.11 \\
05 & 1.8 & 2.78 & 5.1 & 1.0 & 2.08 & 3.24 \\
06 & 2.1 & 1.83 & 2.1 & 1.8 & 3.03 & 3.19 \\
07 & 2.1 & 2.00 & 2.3 & 1.7 & 2.95 & 3.25 \\
08 & 2.1 & 1.87 & 2.4 & 1.7 & 2.92 & 3.25 \\
09 & 2.1 & 1.91 & 2.5 & 1.7 & 2.88 & 3.25 \\
10 & 2.1 & 2.23 & 2.6 & 1.6 & 2.85 & 3.33 \\
11 & 2.1 & 2.02 & 2.9 & 1.6 & 2.78 & 3.33 \\
12 & 2.1 & 2.52 & 3.1 & 1.6 & 2.73 & 3.33 \\
13 & 2.1 & 2.11 & 3.2 & 1.5 & 2.71 & 3.41 \\
14 & 2.1 & 2.38 & 3.7 & 1.4 & 2.63 & 3.50 \\
15 & 2.1 & 2.79 & 4.3 & 1.3 & 2.56 & 3.61 \\
16 & 2.3 & 2.61 & 2.1 & 2.0 & 3.42 & 3.48 \\
17 & 2.3 & 2.77 & 2.3 & 1.9 & 3.32 & 3.54 \\
\hline \hline
\end{tabular}

The cross section at $E_{\text {c.m. }}=17.6 \mathrm{MeV}$, having a small statistical error, plays a major role in our $\chi^{2}$ fits. It is characteristic of all our potentials that the theoretical values of $\sigma(\Lambda p)$ at this energy are too large, and that the inadequacies of potentials with $b$ too large or too small tend to be accentuated here. Table III lists selected $\Lambda-p$ potentials with acceptable fits to $\sigma(\Lambda p)$ at different intrinsic ranges. These potentials are chosen to represent the spread of values of $a_{s} / a_{t}$ consistent with each intrinsic range.

As seen also in Fig. 5, an important distinction between the best fits at different intrinsic ranges is the predicted behavior of $\sigma(\Lambda p)$ at energies below $E_{\text {c.m. }}=3.7 \mathrm{MeV}$. Best fits to $\sigma(\Lambda p)$ generally are characterized by $a_{s} \approx a_{t}$. However, many excellent fits for large values of the ratio $a_{s} / a_{t}$ occur and lead to self-consistent potentials which fit three- and four-body data. It would appear that better $\Lambda-p$ scattering data at lower energies would be helpful in resolving this ambiguity of our analysis.

In using the effective-range approximation with four parameters, Alexander et al. and Secci-Zorn et al. determined the set of parameters shown in Table IV along with an effective-range-parameter search of ours using the combined data. For comparison, our best $\Lambda-p$ potential which also satisfies self-consistency (see Sec. IV) is listed. Lower confidence levels are associated with effective-range fits partly because of a reduction by 1 of the number of degrees of freedom.

As in the effective-range approximation, our three-parameter-potential fit neglects any pos-

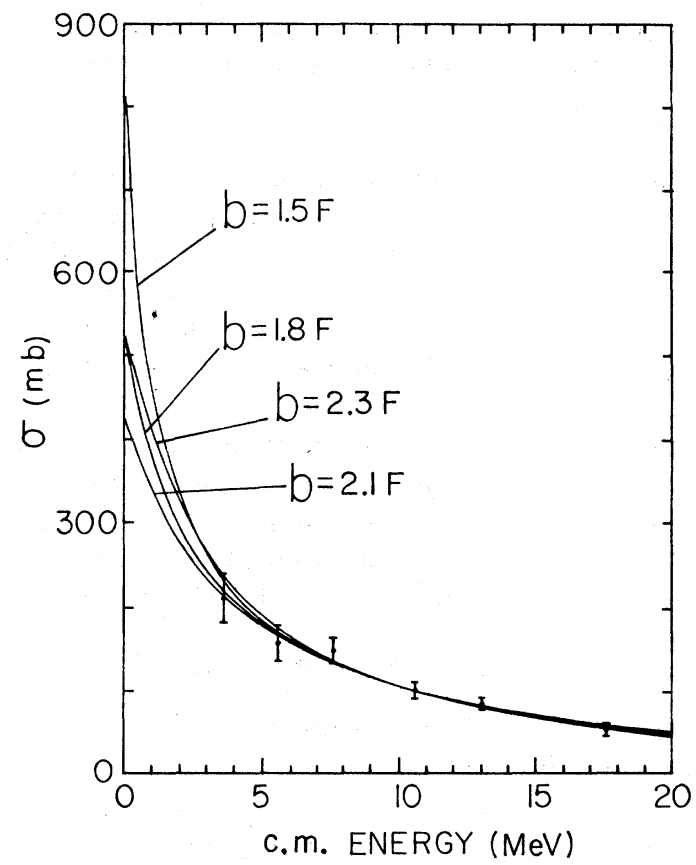

FIG. 5. Best fits to $\sigma(\Lambda p)$ for four intrinsic ranges.

sible $\boldsymbol{P}$-wave contributions to $\sigma(\Lambda p)$. HT have listed $P$-wave phase shifts from their potential $H$ with a hard-core radius $r_{c}=0.6 \mathrm{~F}$, which give a $3 \%$ contribution to $\sigma(\Lambda p)$ at $E_{\text {c.m. }}=17.1 \mathrm{MeV}$. Since we do not have a theory of the $\boldsymbol{P}$-wave interaction, it seems more consistent to ignore such small $P$-wave effects in determination of $\Lambda-p$ potential parameters. It should be mentioned, however, that a check has been made of the effect of subtracting the HT $P$-wave contributions directly from the six data points and using these new data in our scanning procedures. The result is only a slight increase in $\chi^{2}$ for a given set of $\Lambda-p$ parameters found. Furthermore, a strictly attractive $P$-wave potential would be a more appropriate

TABLE IV. Various fits to low-energy $\Lambda-p$ data.

\begin{tabular}{lccccccc}
\hline \hline \multicolumn{1}{c}{ Source } & $\chi^{2}$ & $\begin{array}{c}-a_{t} \\
(\mathrm{~F})\end{array}$ & $\begin{array}{c}-a_{s} \\
(\mathrm{~F})\end{array}$ & $\begin{array}{c}r_{0 t} \\
(\mathrm{~F})\end{array}$ & $\begin{array}{c}r_{0 s} \\
(\mathrm{~F})\end{array}$ & $\begin{array}{c}\text { Confidence }^{\mathrm{a}} \\
\text { level }\end{array}$ \\
\hline Ref. 11 & & 1.6 & 1.8 & 3.3 & 2.8 & \\
Ref. 12 $^{\mathrm{b}}$ & 2.1 & 2.2 & 2.0 & 3.5 & 5.0 & 0.34 \\
${\text { Ref. } 7{ }^{\mathrm{c}}}^{\mathrm{b}}$ & 3.6 & 2.1 & 2.3 & 3.4 & 3.3 & \\
This study & 2.04 & 1.9 & 1.9 & 3.4 & 3.4 & 0.37 \\
Pot. 08 & 1.87 & 1.7 & 2.4 & 3.25 & 2.9 & 0.60 \\
\hline \hline
\end{tabular}

\footnotetext{
${ }^{a}$ Accumulative distribution of $\chi^{2}$.

${ }^{\mathrm{b}}$ Four-parameter effective-range fits to $\sigma(\Lambda p)$. Parameters for this study were found in $\chi^{2}$ search of combined data of Table I.

${ }^{c}$ Pot. $H$ of HT, having hard-core radius $r_{c}=0.6 \mathrm{~F}$ and exponential shape.
} 
extension of our Yamaguchi $S$-wave interaction than the HT hard-core potential. Impact-parameter considerations suggest that such a potential could give lower $P$-wave contributions at $E_{\text {c.m. }}$. ऽ $18 \mathrm{MeV}$.

\section{SELF-CONSISTENT $\Lambda-N$ POTENTIALS}

The $\Lambda-p$ and CS interaction strengths are related by

$$
\begin{aligned}
& \lambda_{s x}=\lambda_{s}+\lambda_{B}(\alpha-3), \\
& \lambda_{t x}=\lambda_{t}+\lambda_{B}(\alpha+1) .
\end{aligned}
$$

It is convenient to combine the relationships involving two- and four-baryon data, Eqs. (17) and (7), obtaining the dependence of $\lambda$ upon $\Delta, M, \lambda_{s x}$, and $\lambda_{t x}$. Defining $\lambda_{x}=\frac{3}{4} \lambda_{s x}+\frac{1}{4} \lambda_{t x}$, we see that the two- and four-body equations combine to give an expression for $\lambda$, which we denote by $\lambda_{2,4}$. For $I=0$ this becomes

$$
\lambda_{2,4}=\frac{\lambda_{x}(1+\Delta)-\lambda_{t x} \Delta}{1+\sqrt{3} \Delta(\sqrt{3}+M)+5 M / \sqrt{3}},
$$

while $I=1$ gives

$$
\lambda_{2,4}=\frac{\lambda_{x}\left(\frac{1}{3}+\Delta\right)+\lambda_{t x}\left(\frac{2}{3}-\Delta\right)}{1+\sqrt{3} \Delta(\sqrt{3}+M)+M / \sqrt{3}} .
$$

Part of the self-consistency problem arises through the different dependences of $\lambda$ upon $M$ given by Eqs. (18a) and (18b) and by the solution of the three-body problem. Given any data fit, represented by $\left(B_{\Lambda}, \Delta, \lambda_{s x}, \lambda_{t x}, b\right)$, the spin dependence in $W_{\text {CSB }}$ must be adjusted so as to give the same value of $\lambda$ from Eq. (18a) or (18b) as the eigenvalue, $\lambda$, in ${ }_{\Lambda} \mathrm{H}^{3}$. We can denote this eigen-

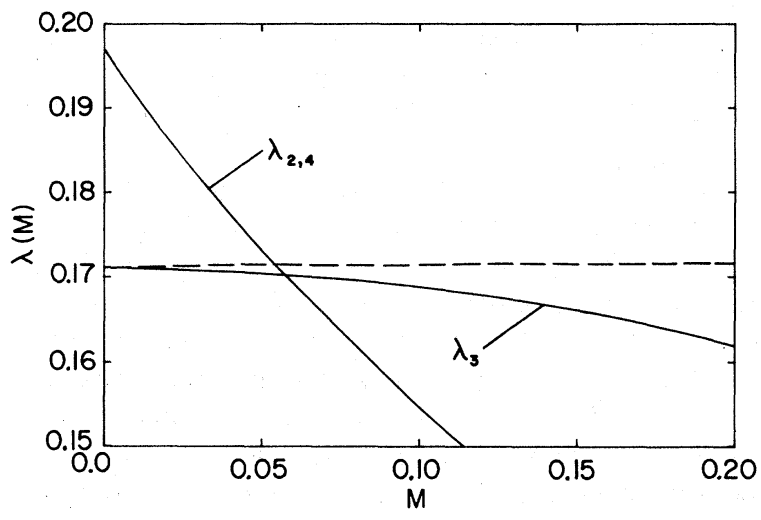

FIG. 6. Graphical illustration of the self-consistent solution of the two-, three-, and four-body problems. $\lambda_{2,4}$ is determined from the two- and four-body data and $\lambda_{3}$ is the eigenvalue from the three-body problem. The dashed line represents $\lambda_{3}$ without inclusion of the $T=1$ state. The point of intersection determines the selfconsistent value of $\lambda\left(\right.$ in $\mathrm{F}^{-3}$ ) and $M$. value by $\lambda_{3}$. A solution is sketched graphically in Fig. 6 which illustrates the considerably stronger $M$ dependence in $\lambda_{2,4}$ than in $\lambda_{3}$. In practice, an iterative procedure is followed starting at $M \gtrless 0$, with successive recomputations of the eigenvalue $\lambda_{3}$ at different values of $M$ until self-consistency is achieved.

Self-consistency requires, in addition, that $S>0$. If one assumes that the CS forces determine the spin of ${ }_{\Lambda} \mathrm{H}^{3}$, then a negative value of $S$ is inconsistent $^{32}$ with the experimental spin value of $\frac{1}{2}$. In terms of the two- and four-baryon data, $S$ is expressible as a decreasing function of $M$. We define an experimental spin-dependence parameter, $S_{x}$ :

$$
S_{x}=\frac{1}{4} \sqrt{3} \lambda^{-1}\left(\lambda_{s x}-\lambda_{t x}\right) \text {. }
$$

$S_{x}$ is the sum of a CS and CSB part, expressed simply as

$$
S_{x}=S+M \text {. }
$$

The inequality $d S / d M<0$ holds for $I=0$ whenever $\lambda_{s x}<3 \lambda_{t x}$ and for $I=1$ whenever $\lambda_{s x}>0$ and $\lambda_{t x}>0$. Hence if $S<0$ at the beginning or the end of an iteration, then the particular data fit must be rejected as unphysical.

Finally, a third self-consistency requirement arises from the spin assignment for ${ }_{\Lambda} \mathrm{He}^{4}$. As is easily seen from Eqs. (7) and (18), the spin assignments (a) $I=0$ and (b) $I=1$ are met self-consistently only if $S>M$ or $M>S$, respectively. These inequalities provide strong conditions which eliminate many fits to $\sigma(\Lambda p)$.

$\Lambda-N$ potentials have been computed for all intrinsic ranges used in fits to $\sigma(\Lambda p)$. In what follows, attention is directed only at the case $b=2.1 \mathrm{~F}$, because this case adequately and concisely illustrates our results. In addition to there being slightly better $\sigma(\Lambda p)$ fits at $b=2.1 \mathrm{~F}$ than any other being considered, we find here the largest spread of self-consistent solutions with respect to the ratio $a_{s} / a_{t}$. Table $\mathrm{V}$ contains all the self-consistent $\Lambda-N$ potentials we find by using the $\Lambda-p$ potentials 06 to 15 of Table III. In Table V, the suffix notation $A, B, C$, refers, respectively, to the cases $B_{\Lambda}=0.05,0.17$, and $0.25 \mathrm{MeV}$. The prefix $S$ denotes the case $I=1$. As Table $V$ tends to suggest, we find no solutions for $I=1$ at a value of $B_{\Lambda}$ greater than $0.05 \mathrm{MeV}$.

Over the entire range of self-consistent fits of all $50 \Lambda-p$ potentials, the $\Lambda-p$ CSB interactions are attractive. Defining $r_{s t}=\epsilon_{s} / \epsilon_{t}$, we find that $1.25 \leqslant r_{s t} \leqslant 2.59$ for $I=0$ and $2.58 \leqslant r_{s t} \leqslant 4.21$ for $I=1$. From Table $\mathrm{V}$ the CSB spin dependence clearly is similar for both spin assignments. However, the values of $M$ seen there lie near the upper limit for $I=0$ and the lower limit for $I=1$ from among the entire classes of self-consistent solu- 
TABLE V. $\Lambda-N$ self-consistent potentials for $b=2.1 \mathrm{~F} .^{a}$

\begin{tabular}{ccccccccc}
\hline \hline Pot. ${ }^{\mathrm{b}}$ & $M$ & \multicolumn{1}{c}{$\begin{array}{c}\lambda \\
\left(\mathrm{F}^{-3}\right)\end{array}$} & $\begin{array}{c}\lambda_{B} \\
\left(\mathrm{~F}^{-3}\right)\end{array}$ & $\alpha$ & $\begin{array}{c}r_{s x} \\
(\mathrm{~F})\end{array}$ & $\begin{array}{c}r_{t x} \\
(\mathrm{~F})\end{array}$ & $r_{s t}$ \\
\hline $10 A$ & 0.0474 & 0.0475 & 0.157 & -0.00430 & -4.07 & 0.158 & 0.0837 & 2.30 \\
$13 A$ & 0.0684 & 0.0771 & 0.157 & -0.00619 & -3.73 & 0.203 & 0.110 & 2.47 \\
$14 A$ & 0.0813 & 0.103 & 0.157 & -0.00734 & -3.60 & 0.226 & 0.129 & 2.54 \\
$15 A$ & 0.0935 & 0.130 & 0.156 & -0.00843 & -3.51 & 0.246 & 0.149 & 2.59 \\
$08 B$ & 0.0175 & 0.0462 & 0.167 & -0.00169 & -5.89 & 0.0805 & 0.0510 & 1.82 \\
$09 B$ & 0.0219 & 0.0490 & 0.167 & -0.00212 & -5.30 & 0.0929 & 0.0563 & 1.93 \\
$10 B$ & 0.0239 & 0.0654 & 0.167 & -0.00212 & -5.09 & 0.0974 & 0.0600 & 1.98 \\
$11 B$ & 0.0355 & 0.0726 & 0.167 & -0.00342 & -4.40 & 0.127 & 0.0738 & 2.18 \\
$12 B$ & 0.0425 & 0.0768 & 0.167 & -0.00409 & -4.17 & 0.144 & 0.0822 & 2.26 \\
$13 B$ & 0.0432 & 0.0936 & 0.167 & -0.00416 & -4.14 & 0.144 & 0.0854 & 2.28 \\
$14 B$ & 0.0550 & 0.118 & 0.167 & -0.00529 & -3.88 & 0.170 & 0.103 & 2.39 \\
$15 B$ & 0.0663 & 0.144 & 0.166 & -0.00637 & -3.72 & 0.192 & 0.122 & 2.47 \\
& & & & & & \\
$07 C$ & 0.00360 & 0.0510 & 0.171 & -0.000360 & -16.9 & 0.0390 & 0.0353 & 1.25 \\
$08 C$ & 0.00810 & 0.0539 & 0.171 & -0.000800 & -9.19 & 0.0523 & 0.0405 & 1.49 \\
$09 C$ & 0.0124 & 0.0567 & 0.171 & -0.00123 & -7.04 & 0.0652 & 0.0459 & 1.66 \\
$10 C$ & 0.0143 & 0.0728 & 0.171 & -0.00141 & -6.49 & 0.0697 & 0.0491 & 1.73 \\
$11 C$ & 0.0255 & 0.0798 & 0.171 & -0.00252 & -4.94 & 0.100 & 0.0630 & 2.01 \\
$12 C$ & 0.0322 & 0.0840 & 0.171 & -0.00319 & -4.53 & 0.118 & 0.0715 & 2.13 \\
$13 C$ & 0.0329 & 0.100 & 0.171 & -0.00326 & -4.49 & 0.119 & 0.0744 & 2.15 \\
$14 C$ & 0.0444 & 0.124 & 0.171 & -0.00438 & -4.09 & 0.145 & 0.0915 & 2.30 \\
$15 C$ & 0.0552 & 0.149 & 0.171 & -0.00544 & -3.86 & 0.167 & 0.109 & 2.40 \\
$S 11 A$ & 0.0744 & 0.0408 & 0.157 & -0.00673 & -2.78 & 0.195 & 0.0759 & 3.25 \\
$S 12 A$ & 0.0944 & 0.0332 & 0.156 & -0.00850 & -2.24 & 0.219 & 0.0672 & 4.21 \\
\hline \hline
\end{tabular}

${ }^{a}$ All self-consistent solutions are shown which are obtained using $\Lambda-p$ potentials 0.06 to 15 (see Table III). The effects of the $T=1$ state in ${ }_{\Lambda} \mathrm{H}^{3}$ are included.

${ }^{\mathrm{b}}$ Suffixes $A, B, C$ denote $B_{\Lambda}=0.05,0.17$, and $0.25 \mathrm{MeV}$, respectively. Prefix $S$ refers to potentials consistent with spin 1 for ${ }_{\Lambda} \mathrm{He}^{4}$.

tions. Hence, knowledge of the spin of ${ }_{\Lambda} \mathrm{He}^{4}$ might serve to delimit the CSB spin dependence.

The dependence of $\lambda_{3}$ upon $M$ is induced through isospin mixing. Although in our model of $\Lambda-N$ interactions $\lambda_{2,4}$ has a more rapid variation with $M$ than does $\lambda_{3}$, the point of intersection of the two curves illustrated in Fig. 6 is shifted significantly from the case of no isospin mixing. Omis sion of $|-, 1\rangle$ would be represented by a horizontal line for $\lambda_{3}$. As one observes in Fig. 6, inclusion of the $|-, 1\rangle$ state in ${ }_{\Lambda} \mathrm{H}^{3}$ has the effect of shifting some of the spin dependence from $U_{\mathrm{CS}}$ to $W_{\mathrm{CSB}}$. This shift of spin dependence can be seen in Table VI, which lists the percentage change of self-consistent potential parameters upon including the $T=1$ state of ${ }_{\Lambda} \mathrm{H}^{3}$. The potentials consistent with lower $B_{\Lambda}$ are clearly the more sensitive to isospin-mixing effects. The most pronounced effects are seen to occur for $I=1$.

To assess the sizes of CSB required in our analysis, it is useful to define CSB fractions $r_{s x}$ and $r_{t x}$ by the equations

$$
\begin{aligned}
& r_{s x}=\epsilon_{s} / \lambda_{s x}, \\
& r_{t x}=\epsilon_{t} / \lambda_{t x} .
\end{aligned}
$$

For $\boldsymbol{B}_{\Lambda}=0.05 \mathrm{MeV}$ and $I=0$, both CSB fractions are increased upon inclusion of $|-, 1\rangle$, whereas for $I=1$, isospin mixing increases $r_{s x}$ but decreases $r_{t x}$, the combination of which reinforces to increase $M$ and decrease $S$. The entry for Pot. $S 12 A$ shows it can sometimes be hazardous to omit isospin mixing in ${ }_{\Lambda} \mathrm{H}^{3}$, if one's goal is to determine either the CS or the CSB $\Lambda-N$ potential parameters self-consistently.

The correlation of the CSB fractions with $B_{\Lambda}$ is graphically illustrated in Fig. 7, for the case $I=0$.

Although $r_{s x}$ and $r_{t x}$ are poorly determined by the data, the smaller values of $\boldsymbol{B}_{\Lambda}$ can clearly lead to a higher value of these fractions. In our model, the $\Lambda-p$ potential is stronger than what is required of a CS potential to bind ${ }_{\Lambda} \mathrm{H}^{3}$, and this difference becomes larger for lower $B_{\Lambda}$. It should be emphasized that $M$ is not a free parameter, in spite of the appearance of $M$ on the abscissa of Fig. 7. $M$ is determined uniquely for any data fit in a self-consistent manner.

\section{DISCUSSION}

From the results of Sec. IV it is clear that the $\Lambda-N$ CSB potential required to fit two-, three-, 
TABLE VI. Effect of isospin mixing in ${ }_{\Lambda} \mathrm{H}^{3} .^{\mathrm{a}}$

\begin{tabular}{lllllllll}
\hline \hline Pot. & $M$ & $S$ & $\lambda$ & $\lambda_{B}$ & $\alpha$ & $r_{s x}{ }^{b}$ & $r_{t x} c^{\prime}$ & $r_{s t}$ \\
\hline $10 A$ & 2.6 & -1.9 & -0.3 & 2.1 & 0.6 & 1.7 & 1.2 & 0.5 \\
$13 A$ & 3.6 & -1.9 & -0.5 & 3.0 & 0.7 & 2.6 & 2.0 & 0.5 \\
$14 A$ & 4.2 & -1.8 & -0.8 & 3.4 & 0.7 & 3.0 & 2.4 & 0.6 \\
$15 A$ & 4.9 & -1.7 & -1.0 & 3.8 & 0.7 & 3.4 & 2.8 & 0.6 \\
$07 C$ & 0 & 0 & 0 & 0 & -1.1 & -0.9 & -1.1 & 0.2 \\
$08 C$ & 0 & -0.2 & 0 & 0 & -0.4 & -0.3 & -0.5 & 0.1 \\
$09 C$ & 0.8 & -0.2 & 0 & 0.8 & -0.5 & 0.5 & 0.3 & 0.2 \\
$10 C$ & 0.7 & -0.1 & 0 & 0.7 & -0.4 & 0.4 & 0.2 & 0.2 \\
$11 C$ & 1.6 & -0.4 & 0 & 1.2 & -0.6 & 0.8 & 0.4 & 0.4 \\
$12 C$ & 1.6 & -0.4 & -0.1 & 1.9 & -0.6 & 1.5 & 1.1 & 0.4 \\
$13 C$ & 1.9 & -0.3 & -0.1 & 1.9 & -0.6 & 1.5 & 1.1 & 0.4 \\
$14 C$ & 2.5 & -0.5 & -0.2 & 2.3 & -0.6 & 1.9 & 1.4 & 0.5 \\
$15 C$ & 3.0 & -0.5 & -0.4 & 2.5 & -0.6 & 2.1 & 1.6 & 0.5 \\
$S 11 A$ & 4.2 & -3.3 & -0.6 & 19.3 & -20.4 & 6.0 & -4.4 & 25.2 \\
$S 12 A$ & 29.1 & -36.8 & -1.3 & 28.4 & -28.0 & 9.0 & -25.3 & 45.2 \\
\hline \hline
\end{tabular}

${ }^{a}$ Change of potential parameters upon inclusion of $T=1$ state, expressed in percent of values obtained when $T=1$ state is not included.

${ }^{\mathrm{b}} r_{\mathrm{sx}}=\epsilon_{\mathrm{x}} / \lambda_{\mathrm{sx}} \cdot{ }^{\mathrm{c}} r_{t x}=\epsilon_{t} / \lambda_{t x}$.

and four-body hypernuclear data can be stronger than what is necessary to explain the experimental CSB effect in ${ }_{\Lambda} \mathrm{He}^{4}$ and ${ }_{\Lambda} \mathrm{H}^{4}$ alone. For $I=0$, the value -3 for the parameter $\alpha$ would represent complete cancellation between the spin-dependent $(\vec{\sigma} \cdot \vec{\sigma})$ and spin-independent $(\alpha)$ contributions to $\Delta$. Cancellations are seen to be large when $I=0$, and are also significant when $I=1$, where CSB must work against the spin dependence of the CS interaction in providing the correct sign of the fourbody energy splitting. The spin assignment $I=1$ is inconsistent with setting $\alpha=0$ and the known experimental spin of 0 for ${ }_{\Lambda} \mathrm{H}^{4}$, if one assumes that

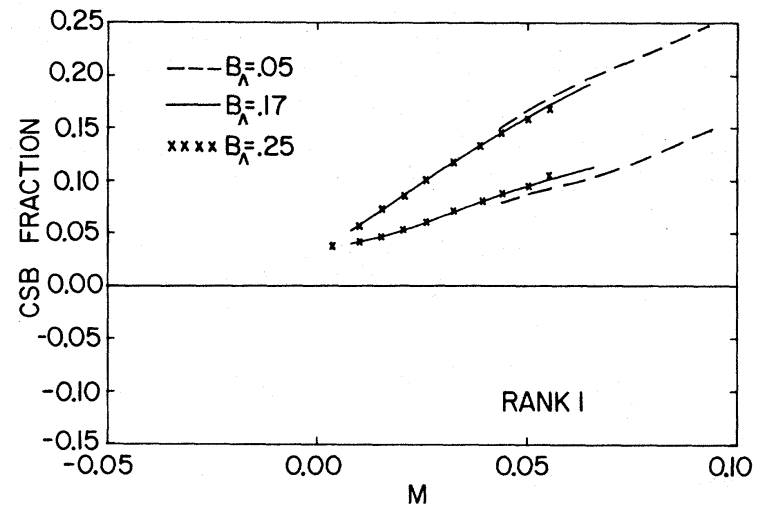

FIG. 7. CSB fractions as a function of the self-consistent value of $M . M$ is the CSB spin-difference parameter. Upper curves are singlet fractions $\left(r_{s x}\right)$ and lower curves are triplet fractions $\left(r_{t x}\right)$ as defined in Eq. (21). These are smooth curves drawn through a plot of all self-consistent CSB potentials in order to show the systematic variation with the assumed values of $B_{\Lambda}$. a two-body central-force model is valid. In the present analysis the spin-independent parameter $\alpha$ plays a vital role in achieving maximum consistency with all the data.

Only five self-consistent solutions have been found for $I=1$, all for $B_{\Lambda}=0.05 \mathrm{MeV}$, and all associated with excellent fits to $\sigma(\Lambda p)$. If the spin of ${ }_{\Lambda} \mathrm{He}^{4}$ is 1 , then our model predicts a strong CSB component.

For most potentials, isospin mixing in ${ }_{\Lambda} \mathrm{H}^{3}$ has the effect of contributing to an increase in the $W_{\text {CSB }}$ found. This is most pronounced for $I=1$. To provide a more complete picture of CSB effects in three-body states, it would seem desirable to study $\Lambda-d$ scattering as well. A large CSB effect might be encountered relative to $\Lambda-p$ scattering, and the CSB coupling of the $T=0$ and $T=1$ channels could be important.

As we have not solved the four-body problem, there is an unknown error associated with our fit of complete four-baryon data. A solution of the four-body problem using separable interactions would be an important step. In addition, definite predictions are desirable with our potential models concerning the existence of particle-stable excited states of ${ }_{\Lambda} \mathrm{He}^{4}$ and ${ }_{\Lambda} \mathrm{H}^{4}$. Such states have been inferred by Herndon and Tang, ${ }^{7,33}$ and experimental evidence has recently been discovered in support of them. ${ }^{34}$

That part of the four-baryon data we have chosen, namely $\Delta$, could be too small, in view of the strictly attractive-potential model employed with its relatively smaller kinetic energies. Because this error is unknown and conceivably large, we 
TABLE VII. Sensitivity to $\Delta$. Percent change of potential parameters when $\Delta$ is increased by $1 \%$, computed as an average over an interval of $50 \%$ increase of $\Delta$.

\begin{tabular}{lcccccccc}
\hline \hline Pot. & $M$ & $S$ & $\lambda$ & $\lambda_{B}$ & $\alpha$ & $r_{s x}$ & $r_{t x}$ & $r_{s t}$ \\
\hline $10 A$ & -0.2 & 0.2 & 0.001 & -0.2 & 0.4 & -0.04 & 0.5 & -0.2 \\
$13 A$ & -0.2 & 0.2 & 0.001 & -0.2 & 0.2 & -0.03 & 0.2 & -0.1 \\
$15 A$ & -0.1 & 0.1 & 0.003 & -0.1 & 0.2 & -0.03 & 0.1 & -0.1 \\
$08 B$ & -0.6 & 0.2 & 0.000 & -0.6 & 1.0 & -0.07 & 0.4 & -0.4 \\
$13 B$ & -0.2 & 0.1 & 0.001 & -0.2 & 0.4 & -0.04 & 0.3 & -0.4 \\
$15 B$ & -0.2 & 0.07 & 0.001 & -0.2 & 0.2 & -0.03 & 0.1 & -0.2 \\
\hline \hline
\end{tabular}

have made a test of sensitivity to $\Delta$. Table VII shows the result of this test, applied to the case $I=0$. All parameters, with the exception of $\alpha$, are seen to be fairly insensitive to changes of $\Delta$ by a few percent. The singlet CSB fraction $r_{s x}$ is particularly stable with respect to variation of $\Delta$.

A possible spin- $\frac{3}{2}$ bound state of ${ }_{\Lambda} \mathrm{H}^{3}$ has been sought with all our $\Lambda-N$ potentials. Considering only the dominant state in ${ }_{\Lambda} \mathrm{H}^{3}$, with its pure triplet CS interaction, leads to minimum CS $\Lambda-N$ triplet strengths (in $\mathrm{F}^{-3}$ ) for a spin- $\frac{3}{2}$ bound state of $0.454,0.249$, and 0.158 , corresponding to intrinsic ranges (in F) of $1.5,1.8$, and 2.1, respectively. None of the potentials constructed with the assumption $I=1$ lead to such a bound state. For $I=0$, one potential with a spin $-\frac{3}{2}$ bound state occurs at $B_{\Lambda}=0.25 \mathrm{MeV}$, with $b=2.1 \mathrm{~F}$, and $\chi^{2}=2.70$. The scattering lengths for this potential are $a_{s}=-2.4 \mathrm{~F}$, $a_{t}=-1.8 \mathrm{~F}$. The existence in our model of spin- $\frac{3}{2}$ states is not very sensitive to $\Delta$, since as $\Delta$ increases, both $S$ and $\lambda$ increase, with canceling effects upon $\lambda_{t}=\lambda(1-\sqrt{3} S)$.

We find no $\Lambda-N$ bound states. The well-depth parameters are the numbers by which singlet and triplet interaction strengths must be divided to produce just-bound states. Typical $\Lambda-p$ and $\Lambda-n$ singlet and triplet values are $0.6-0.7$ or less. Hence in this sense our analysis leads to generally weaker $\Lambda-N$ interactions than the best potential of $\mathrm{HT}$, with $\Lambda-p$ singlet and triplet values of 0.8 .

Figure 7 reveals the inconsistency between present results and the $\mathrm{SU}_{3}$ particle-mixing theory of CSB of Downs. At no point does the singlet CSB fraction become negative, which would correspond to a $\Lambda-p$ CSB repulsion. A parallel study to the present paper in which more realistic $\Lambda-N$ and $N-N$ potentials with short-range repulsions are used is discussed in the following paper, and shows the dependence of this conclusion upon our assumptions concerning the shape of $\Lambda-N$ and $N-N$ interactions.

\section{ACKNOWLEDGMENTS}

We have received important comments from J. S.
Levinger, M. Fuda, E. Harms, and R. Stagat concerning the three-body problem. We are also grateful to G. Alexander, J. Brown, A. Gal, J. Sacton, and J. Schneps for helpful discussions. One of us (K. H.) wishes to thank Y. C. Tang for communicating results before publication and A. Bodmer for some stimulating discussions about hypernuclei during the author's visit to Argonne National Laboratory.

\section{APPENDIX}

Specializing to the case where $|+, 1\rangle$ and $|-, 0\rangle$ are neglected, we write the momentum-space wave function of ${ }_{\Lambda} \mathrm{H}^{3}$

$$
\Psi=\varphi|+, 0\rangle+\gamma|-, 1\rangle .
$$

The $T=0$ and $T=1$ amplitudes $\varphi$ and $\gamma$ satisfy coupled integral equations obtained by substituting Eqs. (10) into the Schrödinger equation

$$
\Psi=-D^{-1} V_{\text {tot }} \Psi
$$

and employing NLS potentials, as defined in Eq. (4), for $\Lambda-N$ and $N-N$ interactions. Here $D=T+E$, $T$ and $V_{\text {tot }}$ are the total kinetic- and potentialenergy operators, and $E$ is the total binding energy. The operator $-D^{-1} V_{\text {tot }}$ is not Hermitian. This is an artifact of the specific manner in which the Schrödinger equation is employed, as occurs similarly with the variation-iteration method for determining interaction strengths when energies are assumed to be given. ${ }^{35}$ As a consequence, the numerical eigenvalue problem finally obtained involves a nonsymmetric matrix. With the $n-p$ interaction and $E$ fixed, our procedure is to look for the minimum over-all $\Lambda-N$ interaction strength, $\lambda$.

The calculation is performed in the c.m. system, in which $\overrightarrow{\mathrm{P}}_{1}=-\left(\overrightarrow{\mathrm{P}}_{2}+\overrightarrow{\mathrm{P}}_{3}\right)$, and using $\overrightarrow{\mathrm{P}}_{2}$ and $\overrightarrow{\mathrm{P}}_{3}$ as the independent momenta. Then $D=D\left(P_{2}, P_{3}, E\right)$. We write the $n$ - $p$ singlet and triplet kernels as $f_{s}(P)$ and $f_{t}(\boldsymbol{P})$. Taking all $\Lambda-N$ kernels to be of the same form, written $g(P)$, leads to the structure

$$
\begin{aligned}
& \varphi=\frac{1}{D}\left[f_{t}(y) \frac{A(x)}{x}+g(\bar{P}) \frac{B(N)}{N}+g(\bar{N}) \frac{B(P)}{P}\right], \\
& \gamma=\frac{1}{D}\left[f_{s}(y) \frac{C(x)}{x}+g(\bar{P}) \frac{H(N)}{N}+g(\bar{N}) \frac{H(P)}{P}\right],
\end{aligned}
$$

where

$$
\begin{aligned}
& \overrightarrow{\mathrm{x}}=-\overrightarrow{\mathrm{P}}_{1}, \quad x=|\overrightarrow{\mathrm{x}}|, \quad \overrightarrow{\mathrm{y}}=\frac{1}{2}\left(\overrightarrow{\mathrm{P}}_{3}-\overrightarrow{\mathrm{P}}_{2}\right), \\
& \overrightarrow{\mathrm{P}}=\frac{\mu \overrightarrow{\mathrm{P}}_{2}-M \overrightarrow{\mathrm{P}}_{1}}{\mu+M}=\overrightarrow{\mathrm{P}}_{2}+\frac{1}{2} R \overrightarrow{\mathrm{P}}_{3}, \\
& \overrightarrow{\mathrm{N}}=\frac{\mu \overrightarrow{\mathrm{P}}_{3}-M \overrightarrow{\mathrm{P}}_{2}}{\mu+M}=\overrightarrow{\mathrm{P}}_{3}+\frac{1}{2} R \overrightarrow{\mathrm{P}}_{2} .
\end{aligned}
$$


$M$ and $\mu$ are mean nucleon and $\Lambda$ masses, and $R=2 M /(\mu+M)$.

The spectator functions, $A, B, C$, and $H$, satisfy four coupled integral equations. Upon substituting (A3) into the Schrödinger equation, one obtains

$$
\begin{gathered}
A(x)=\frac{\lambda_{t e}}{M}\left[F_{t}(x) A(x)+2 \int_{0}^{\infty} d z J_{1}(x, z) B(z)\right], \\
B(N)=\frac{\lambda_{2}}{R \mu}\left[G(N) B(N)+\int_{0}^{\infty} d z J_{1}(z, N) A(z)\right. \\
\left.\quad+\int_{0}^{\infty} d z J_{3}(N, z) B(z)\right] \\
+\frac{\sqrt{3} \epsilon_{\sigma}}{4 R \mu}\left[\int_{0}^{\infty} d z J_{2}(z, N) C(z)+G(N) H(N)\right. \\
\left.+\int_{0}^{\infty} d z J_{3}(N, z) \boldsymbol{H}(z)\right],
\end{gathered}
$$

where $\lambda_{t e}$ is the triplet-even $n-p$ interaction strength and $\lambda_{2}=2 \lambda$. With the notation $u=\cos \theta_{z}$, the kernels are

$$
\begin{aligned}
& J_{1}(z, N)=2 \pi z N \int_{-1}^{+1} d u \frac{g\left(\left|\overrightarrow{\mathrm{z}}+\frac{1}{2} \overrightarrow{\mathrm{P}}_{3}(R-2)\right|\right) f_{t}\left(\left|\overrightarrow{\mathrm{P}}_{3}-\frac{1}{2} \overrightarrow{\mathrm{Z}}\right|\right)}{D\left(\left|\overrightarrow{\mathrm{z}}-\overrightarrow{\mathrm{P}}_{3}\right|, P_{3}\right)}, \\
& J_{2}(z, N)=\left.J_{1}(z, N)\right|_{f_{t} \rightarrow f_{s}}, \\
& J_{3}(z, N)=2 \pi z N \int_{-1}^{+1} d u \frac{g\left(\left|\overrightarrow{\mathrm{z}}+\frac{1}{2} R \overrightarrow{\mathrm{P}}_{3}\right|\right) g\left(\left|\overrightarrow{\mathrm{P}}_{3}+\frac{1}{2} R \overrightarrow{\mathrm{Z}}\right|\right)}{D\left(z, P_{3}\right)}
\end{aligned}
$$

and

$$
\begin{aligned}
& F_{s, t}(x)=\int d^{3} z \frac{f_{s, t}{ }^{2}(z)}{D\left(\left|\frac{1}{2} \overrightarrow{\mathrm{x}}-\overrightarrow{\mathrm{z}}\right|,\left|\frac{1}{2} \overrightarrow{\mathrm{x}}+\overrightarrow{\mathrm{z}}\right|\right)}, \\
& G(N)=\int d^{3} z \frac{g^{2}\left(\left|\overrightarrow{\mathrm{z}}+\frac{1}{2} R \overrightarrow{\mathrm{P}}_{3}\right|\right)}{D\left(z, P_{3}\right)} .
\end{aligned}
$$

Equations for $C(x)$ and $H(N)$ are obtained from (A4) by making the interchanges $\left(s, t, A, B, \lambda_{t e}, \lambda_{2}\right)$ $\rightarrow\left(t, s, C, H, \lambda_{s e}, \bar{\lambda}\right)$. Although $J_{3}$ is a symmetric kernel, $J_{1}$ is not. The $\Lambda$ spectator functions, $A$ and $C$, are readily eliminated between the four equations, leaving two coupled equations containing only symmetric integral operators. The equations are solved with high numerical precision using ten-point Gauss-Gegenbauer quadrature formulas. ${ }^{36}$
†Work supported in part by a grant from the URI Research Committee through a faculty fellowship held by the first author.

*Eppley Foundation for Research Scholar.

${ }^{1} \mathrm{G}$. Bohm et al., Nucl. Phys. B4, 511 (1968).

${ }^{2}$ R. H. Dalitz and F. von Hippel, Phys. Letters $\underline{10}, 153$ (1964), henceforth referred to as DV.

${ }^{3}$ S. Coleman and S. L. Glashow, Phys. Rev. Letters $\underline{6}$, 423 (1961).

${ }^{4}$ B. W. Downs, Nuovo Cimento $43 \mathrm{~A}, 454$ (1966).

${ }^{5}$ L. Lovitch, S. Rosati, and R. H. Dalitz, Nuovo'Cimento 53A, 301 (1968); 53A, 1060 (1968).

${ }^{6}$ A. Deloff, Fortschr. Physik 17, 129 (1969).

${ }^{7}$ R. C. Herndon and Y. C. Tang, Phys. Rev. 159,853 (1967), hereafter referred to as HT. In their calculation HT used a slightly larger value, $0.29 \mathrm{MeV}$, for $\Delta B_{\Lambda}$.

${ }^{8} \mathrm{~B}$. W. Downs and R. J. N. Phillips, Nuovo Cimento $41 \mathrm{~A}, 375$ (1966), hereafter referred to as DP.

${ }^{9} \mathrm{R}$. H. Dalitz, in Proceedings of the International Conference on Hypernuclear Physics, Argonne National Laboratory, 1969, edited by A. R. Bodmer and L. G. Hyman (Argonne National Laboratory, Argonne, Illinois, 1969), Vol. 1, p. 708.

${ }^{10}$ A. N. Mitra, Nucl. Phys. 32 , 529 (1962); J. H. Hetherington and L. H. Schick, Phys. Rev, 139, B1164 (1965).

${ }^{11}$ G. Alexander, U. Karshon, A. Shapira, G. Yekutieli,

R. Engelmann, H. Filtuth, and W. Lughofer, Phys. Rev. 173,1452 (1968).

${ }^{12}$ B. Secci-Zorn, B. Kehoe, J. Twitty, and R. I. Burnstein, Phys. Rev. 175, 1735 (1968).

${ }^{13}$ Value determined (see Ref. 1) from the two-body pionic decay of ${ }_{\Lambda} \mathrm{H}^{3}$. This is close to the mean value of $0.06 \pm 0.06 \mathrm{MeV}$ also obtained in Ref. 1 .

${ }^{14} \mathrm{G}$. Keys et al., Phys. Rev. D 1,67 (1970).
${ }^{15}$ L. H. Schick and A. J. Toepfer, Phys. Rev. 170,946 (1968).

${ }^{16}$ A. R. Bodmer, Phys. Rev. 141, 1387 (1966).

${ }^{17} \mathrm{~J}$. Borysowicz and J. Dabrowski, Phys. Letters 24B, 549 (1967).

${ }^{18}$ Y. Yamaguchi, Phys. Rev. 95, 1628 (1954).

${ }^{19} \mathrm{HT}$ express their proposed potential (Pot. $H$ ) in terms of well depths, which we take as proportional to interaction strengths in evaluating $\Delta$. Their CS and CSB potentials, like ours, have identical shapes.

${ }^{20}$ S. Ali, M. E. Grypeos, and L. P. Kok, Phys. Letters 24B, 543 (1967).

${ }^{21}$. Deloff, Nucl. Phys. B4, 585 (1968).

${ }^{22}$ L. D. Buxton and R. Schrils, Nuovo Cimento $\underline{59 B}, 218$ (1969).

${ }^{23}$ I. E. McCarthy, Introduction to Nuclear Theory (Wiley, New York, 1968).

${ }^{24}$ B. W. Downs and R. H. Dalitz, Phys. Rev. 114, 593 (1959) ; see also R. H. Dalitz, in Interaction of HighEnergy Particles with Nuclei, International School of Physics "Enrico Fermi," Course 38, edited by T. E. O. Ericson (Academic, New York, 1967), pp. 89-131.

${ }^{25}$ J. T. Brown, B. W. Downs, and C. K. Iddings, Ann. Phys. (N.Y.) 60, 148 (1970); B. W. Downs; Ref. 9, p. 51. ${ }^{26}$ A. J. Toepfer and L. H. Schick, Phys. Rev. 175,1253 (1968).

${ }^{27}$ Y. Nogami, Ref. 9, p. 244; Y. Nogami and E. Satoh, Nucl. Phys. B19, 93 (1970); E. Satoh and Y. Nogami, Phys. Letters 32B, 243 (1970).

${ }^{28}$ A. R. Bodmer and D. M. Rote, Ref. 9, p. 521.

${ }^{29} \mathrm{M}$. Verde, in Handbuch der Physik edited by S. Flügge (Springer, Berlin, 1957), Vol. 39, pp. 144-177.

${ }^{30}$ See note added in proof, A. R. Bodmer, in High Energy 
Physics and Nuclear Structure, edited by G. Alexander (North-Holland, Amsterdam, 1967), pp. 60-89.

${ }^{31}$ We employ a definition of scattering length, $a$, and effective range, $\mathrm{r}_{0}$, which is consistent with $k \cot \delta \approx$ $-a^{-1}+\frac{1}{2} r_{0} k^{2}$.

${ }^{32}$ R. C. Herndon, Y. C. Tang, and G. W. Schmid, Phys. Rev. 137, B294 (1965).

${ }^{33}$ Y. C. Tang, Ref. 9, p. 276.
${ }^{34}$ B. Povh II, invited talk at the New York meeting of the American Physical Society, 1971 (unpublished).

${ }^{35}$ P. M. Morse and H. Feshbach, Methods of Theoretical Physics, Part II (McGraw-Hill, New York, 1953), p. 1137.

${ }^{36}$ We are grateful to Dr. J. S. Levinger and Dr. R. Stagat for furnishing us with information about the application of this method.

\title{
$\Lambda$-Nucleon Charge-Symmetry-Breaking Interaction. II. Rank-2 Potentials
}

\author{
E. Sullivan* and K. Hartt \\ Department of Physics, University of Rhode Island, Kingston, Rhode Island 02881 \\ (Received 8 September 1969; revised manuscript received 17 May 1971)
}

\begin{abstract}
A potential model of the $\Lambda-N$ interaction containing short-range repulsions is constructed to study charge-symmetry-breaking (CSB). Rank-2 nonlocal central potentials and a rank-1 approximation to them (referred to as UPAA) are required to fit low-energy $\Lambda-p$ scattering cross sections, the $\Lambda$-separation energy $\left(B_{\Lambda}\right)$ from ${ }_{\Lambda} \mathrm{H}^{3}$, and a dimensionless parameter which partially characterizes the CSB part of the binding-energy difference between ${ }_{\Lambda} \mathrm{H}^{4}$ and ${ }_{\Lambda} \mathrm{He}^{4}$. In a scan of these data, a class of potentials with acceptable $\chi^{2}$ fits is found. Repulsions are seen to reduce significantly the lower limits of the singlet and triplet CSB strengths required in the entire data scan, from the CSB found earlier without including repulsions. In particular, for the largest value of $B_{\Lambda}$ used, $0.25 \mathrm{MeV}$, qualitative agreement with Downs's $\mathrm{SU}_{3}$ model of CSB is attained, in that the $\Lambda-p$ CSB singlet interaction can be repulsive. This conclusion also holds for the UPAA, with an indication that a short-range weakening of $\Lambda-N$ attraction has an effect similar to a $\Lambda-N$ repulsion. Effects of isospin mixing in ${ }_{\Lambda} \mathrm{H}^{3}$ are taken into account. The $\Lambda-N$ potentials resulting from this analysis are seen to vary in a systematic way within the statistical spread of available data.
\end{abstract}

\section{INTRODUCTION}

In the preceding paper ${ }^{1}$ (henceforth referred to as I), we have sought limitations upon the chargesymmetry-breaking (CSB) component of the $\Lambda-N$ interaction as imposed by experiment. Adopting the notation of $\mathrm{I}$, the $\Lambda-N$ two-body CSB interaction is written

$$
W_{C S B}=-\tau_{3}(N)\left(\lambda_{B} / 2 \mu_{\Lambda N}\right)[\alpha+\vec{\sigma}(\Lambda) \cdot \vec{\sigma}(N)] W_{p},
$$

where $\tau_{3}(N)$ is a Pauli isospin operator, $\lambda_{B}$ is an over-all interaction strength, $\alpha$ is a spin-independent constant, and $\mu_{\Lambda N}$ is the $\Lambda-N$ reduced mass. $W_{p}$ contains the momentum dependence of the potential, and is defined to be positive in the limit of low relative momenta. The singlet and triplet CSB strength $s$ in the $\Lambda-p$ state, denoted by $\epsilon_{s}$ and $\epsilon_{t}$, are $\epsilon_{s}=\lambda_{B}(\alpha-3)$ and $\epsilon_{t}=\lambda_{B}(\alpha+1)$. Using two-, three-, and four-baryon data, and a rank-1 centralseparable-potential model in which all potentials, including $W_{p}$, have Yamaguchi shape, ${ }^{2}$ we found in I a class of potentials which gave good $\chi^{2}$ fits to all data. One of our results was to find only attractive $\Lambda-p$ CSB contributions present, expressed by the inequalities $\epsilon_{s}>0, \epsilon_{t}>0$. This disagrees with one of the predictions of the $\mathrm{SU}_{3}$ particle-mixing model of Downs, ${ }^{3}$ that the long-range part of the $\Lambda-p$ CSB singlet interaction should produce a repulsion.

The purpose of the present paper is to test the conclusions of I by extending the analysis to a class of potentials with different shapes. In order to study the effect of short-range repulsions in the $\Lambda-N$ system, we introduce rank-2 interactions. As seen in Secs. II and III, we are thereby able to produce a change in sign of the $S$-wave phase shifts both in the $\Lambda-N$ and the $N-N$ interactions, which is indicative of strong short-range repulsions, and come to better agreement with some of the well-known experimental results for the $\mathrm{N}-\mathrm{N}$ interaction.

Potentials that have a two-body state which is just bound or just unbound are frequently well approximated in the low-energy regime by rank-1 potentials, in what has come to be known as the unitary pole approximation (UPA). ${ }^{4,5}$ We introduce here a closely related approximation, which we refer to as the UPAA. We have in mind the dual purpose of testing the pole approximation to the rank-2 potentials introduced here and studying 